\title{
Atmospheric Effects of Nuclear War Aerosols in General Circulation Model Simulations: Influence of Smoke Optical Properties
}

\author{
Starley L. Thompson and V. Ramaswamy \\ National Center for Atmospheric Research, Boulder, Colorado
}

Curt Cover ${ }^{2}$

Meteorology and Physical Oceanography Dzvzston, Rosenstiel School of Marine and Atmospheric Science, Unsversity of Miami, Miarri, Florida

\begin{abstract}
A global atmospheric general circulation model (GCM) is modified to include radiative transfer parameterizations for the absorption and scattering of solar radiation and the absorption of thermal infrared (IR) radiation by smoke aerosols. The solar scattering modifications include a parameterization for diagnosing smoke optical properties as a function of the time- and spacedependent smoke particle radii. The aerosol IR modifications allow for both the "grey" absorber approximation and a broadband approximation that resolves the aerosol absorption in four spectral intervals. We examine the sensitivity of some GCM-simulated atmospheric and climatic effects to the optical properties and radiative transfer parameterizations used in studies of massive injections of smoke. Specifically, we test the model response to solar scattering versus nonscattering smoke, variations in prescribed smoke single scattering albedo and IR specific absorption, and interactive versus fixed smoke optical properties. Hypothetical nuclear war created smoke scenarios assume the July injection of 60 or $180 \mathrm{Tg}$ of smoke over portions of the mid-latitude land areas of the northern hemisphere. Atmospheric transport and scavenging of the smoke are included. Nonscattering smoke cases produce roughly $40 \mathrm{Wm}^{-2}$ more Earth-atmosphere solar irradiance absorption over the northern hemisphere, when compared to scattering smoke cases having equivalent specific absorption efficiencies. Varying the elemental carbon content of smoke over a plausible range produces a $4^{\circ}-6^{\circ} \mathrm{C}$ change in average mid-latitude land surface temperature, and a variation of about 0.1 in zonally averaged planetary albedo in the northern hemisphere. The inclusion of IR absorption by smoke (IR specific absorption to visible specific extinction ratio of 0.1 ) produces mid-latitude July temperature decreases that are $4^{\circ}-6^{\circ} \mathrm{C}$ smaller in magnitude than produced by IR-transparent cases. Thus the smoke IR opacity effect can make a substantial relative change in land surface temperature estimates when compared to July mid-latitude land temperature decreases of $15^{\circ}-20^{\circ} \mathrm{C}$ found in IR-transparent cases.
\end{abstract}

\section{INTRODUCTION}

The atmospheric effects of smoke aerosols produced in a nuclear war have become a topic of scientific study in the last few years. After the first calculation by Crutzen and Birks [1982] that massive amounts of smoke could be generated by a nuclear war, Turco et al. [1983] produced the first simulation of potential atmospheric and climatic effects using a one-dimensional radiative-convective model. The Turco et al. [1983] model included a rather comprehensive description of atmospheric radiative transfer including solar scattering and thermal infrared (IR) absorption by smoke and nuclear dust aerosols. Later radiative-convective models also incorporated comprehensive radiative transfer descriptions [e.g., Ramaswamy and Kiehl, 1985] and supported the radiative effects found by Turco et al. Although capable of accurately examining the radiative effects of

\footnotetext{
${ }^{1}$ Now at the Program in Atmospheric and Oceanic Sciences, Princeton, New Jersey.

${ }^{2}$ Now at Lawrence Ljvermore National Laboratory, Livermore, California.
}

Copyright 1987 by the American Geophysical Union.

Paper number 7D0519.

0148-0227/87/007D-0519\$05.00 idealized, hemispherically homogeneous aerosol scenarios, radiative-convective model simulations cannot simulate the transient, geographically dependent climatic effects of regional injections of aerosols. For example, the mitigating effects of oceans on land temperatures, the influence of seasons, and the transport of smoke from source regions, cannot be simulated in one-dimensional models.

Initial studies with three-dimensional general circulation models (GCMs) used prescribed, zonally symmetric smoke clouds. Thus these models allowed for the effect of aerosol heating on atmospheric circulation but did not allow for smoke transport and removal [Aleksandrov and Stenchikov, 1983; Covey et al., 1984, 1985; Thompson et al., 1984]. These preliminary GCM studies negelected the scattering of sunlight and absorption of IR by smoke; i.e., smoke was assumed to be purely absorbing at solar wavelengths and transparent at IR wavelengths. While these assumptions are reasonable first approximations for typical carbonaceous smoke, the preliminary studies noted, among other things, the need to improve the GCM treatment of radiative transfer through an atmosphere containing large concentrations of optically absorbing and scattering aerosols.

Cess et al. [1985] incorporated solar scattering by smoke and dust in a two-level GCM but retained the prescription of immobile, IR-transparent aerosol clouds. Their simu- 
lations indicated that land surface temperature reductions created under smoke clouds would be somewhat sensitive to the assumed smoke optical properties. In particular, mid-latitude land temperature decreases under a smoke cloud of extinction optical depth $\tau_{e}=3$ were roughly $5^{\circ} \mathrm{C}$ more for a smoke single scattering albedo of 0.5 as compared to an albedo of 0.7 .

Subsequent GCM studies have relaxed the restrictions on smoke transport. MacCracken and Walton [1984], Ghan et al. [1985, S. J. Ghan, M. C. MacCracken, and J. J. Walton, The climatic response to large atmospheric smoke injections: Sensitivity studies with a tropospheric general circulation model, submitted to the Journal of Geophysical Research, 1987; hereafter called Ghan et al., submitted manuscript, 1987], Thompson [1985] and Malone et al. [1985, 1986] have incorporated smoke as an interactive "tracer" that is transported by large-scale, model-resolved motions. Except for Thompson [1985], each of these studies also included interactive parameterizations of smoke scavenging. These studies have supported the idea that smoke heating could thermally stabilize the middle to upper troposphere and distort normal northern hemispheric atmospheric circulation to such an extent that smoke would tend to be lofted to stratospheric altitudes and transported, in part, toward the southern hemisphere. Moreover, seasonal sensitivity tests have shown that these effects would be most pronounced in summer, when potential solar heating is largest. MacCracken and Walton [1984], and Ghan et al. [1985] included the scattering of solar radiation by smoke in their two-level GCM simulations, but retained the IR-transparent aerosol approximation, and concentrated their analyses on aerosol transport, scavenging and effects on the global hydrologic cycle. Subsequent simulations reported by Ghan et al. (submitted manuscript, 1987) have included the IR effect of smoke.

The purpose of the current study is to examine the sensitivity of some GCM-simulated atmospheric and climatic effects to the optical properties and radiative transfer parameterizations used in studies of massive injections of aerosols. We first describe enhancements to a standard GCM that allows for scattering of solar radiation and absorption of thermal infrared radiation by interactively transported distributions of aerosols. The solar scattering modifications include a parameterization for diagnosing smoke optical properties as a function of the time- and space-dependent smoke particle radii. The aerosol IR modifications allow for both the "grey" absorber approximation and a broadband approximation that resolves the aerosol absorption in four spectral intervals. Simulations are then described that test the model sensitivity to scattering versus nonscattering cases, variations in prescribed smoke single scattering albedo and IR specific absorption, and interactive versus fixed smoke optical properties. We conclude by discussing the general utility of models having an interactive aerosol transport/radiation capability and describing further model enhancements we believe are needed for more reliable simulations of the atmospheric effects of nuclear-war-generated aerosols.

\section{The Model}

The basic character of the GCM used in this study, the NCAR Community Climate Model (CCM), is documented in a series of NCAR technical reports Washington, 1982; Williamson, 1983]. Model modifications of relevance to this study are summarized below and detailed in Appendices $A$ and $B$. The CCMOB version of the model that we use has prescribed diurnally averaged insolation, sea surface temperatures, ozone, land surface properties, and sea ice. In this study these quantities are set equal to their observed climatological average values for July ("perpetual July" mode). The atmosphere and surface are represented by $\sim 4.5^{\circ}$ latitude and $7.5^{\circ}$ longitude resolution, with nine layers throughout the troposphere and stratosphere from the surface to $\sim 30 \mathrm{~km}$ altitude. The CCM produces generally reasonable simulations of temperature and wind fields under normal January and July conditions [Pitcher et al., 1983] or for simulations of the complete seasonal cycle [Washington and Meehl, 1984; Chervin, 1986].

\subsection{Aerosol Transport and Scavenging}

Parameterizations for the transport and scavenging of aerosols are discussed in detail by $\mathrm{S}$. L. Thompson and F. Giorgi, (Atmospheric effects of nuclear war aerosols in GCM simulations: Modeling of smoke microphysical processes, submitted to the Journal of Geophystcal Research, 1987; hereafter referred to as Thompson and Giorgi, submitted manuscript, 1987). The model version used in this study includes three three-dimensional tracer fields that can be fully interactive with model physical processes. Two of the fields are used for smoke; the remaining field is used for dust. The aerosol fields are expressed as lognormal particle size distributions. Thus three parameters are needed at each model grid point to fully describe an aerosol field: the total particle number concentration $N$, the particle geometric mean radius $r_{g}$, and the standard deviation about the geometric mean radius $\sigma_{g}$. In the preliminary tracer simulations described here, the smoke $\sigma_{g}$ is fixed, leaving only two free parameters to describe the size distribution. In practice, the smoke volume concentration $V$ (in cubic meters per kilogram) and $N$ (in number per cubic meters) are carried as the two smoke tracer fields. The radius $r_{g}$ is diagnosed from these two fields and can be used to parameterize optical properties of the smoke as a function of time and place. In cases that include dust, both $\sigma_{g}$ and $r_{g}$ are fixed, leaving the dust volume concentration as the only variable. The simplifying assumption of a lognormal size distribution is, of course, only an approximate representation of the size distribution of aerosols in the present atmosphere. This assumption should be verified in the future for massive smoke injections by detailed aerosol size distribution calculations.

Scavenging of aerosols by wet removal, sedimentation, dry deposition, and coagulation have been incorporated in the modified model. In the simulations reported here the wet removal assumes a hygroscopic aerosol that is removed as a function of the model's liquid water removal rate. Wet removal is responsible for most of the atmospheric removal of aerosol mass in our simulations. Sedimentation and dry deposition are relatively slow processes, given the smoke and dust particle sizes considered here, and consequently, have very little influence on our current results. Coagulation is intraspecific (i.e., smoke particles coagulate with each other, but not with dust that may be 
present) and has a significant effect on the time evolution of mean smoke radii.

The aerosol fields are transported in the model in the same way as water vapor [Williamson, 1983]. The spectral advection scheme used in the CCM is not well suited to tracer studies and is augmented here to correct problems of negative concentrations and rapid dispersal of small positive concentrations. A correction procedure is adopted that tends to damp "small" negative or positive values of tracer concentrations to zero, while leaving the main tracer mass unaffected. Small concentrations are those less than a threshold value that is height and time dependent and is defined as $10 \%$ of the maximum tracer concentration at each vertical level of the model. The tracer fields are multiplied at each model time step by a damping factor that is 0.9 for negative concentrations, and 1.0 for concentrations above the threshold (i.e., no damping). The damping factor varies linearly with concentration between the two limiting cases. This ad hoc procedure sucessfully controls noise but does not guarantee conservation. As a final step, the global tracer concentrations are corrected by multiplying them at every grid point by the ratio of a measure of global tracer amount before and after each threshold damping procedure. This final step guarantees mass conservation by the ad hoc correction procedure but does not correct for mass conservation errors in the standard part of the CCM advection process.

The modified and original model transport schemes were compared to be certain that the modifications had little effect on the bulk movement of a smoke tracer. The modified transport scheme we use compares well with other, more accurate schemes that have been used to advect optically active smoke |Ghan et al., 1985; Malone et al., 1985, 1986] on time scales of about 1 month. However, the standard CCM advection scheme is known to be deficient for long-term transport simulations because of its poor conservation properties and a stratospheric removal rate that is probably too rapid ( $R$. Malone, personal communication, 1986). A more appropriate transport formulation will be included in future longterm simulations, but the present formulation is certainly sufficient to support the conclusions reached in this study (i.e., sufficient for simulations of a few weeks duration based on injections of many teragrams of smoke).

\subsection{Solar Radiative Transfer}

The standard solar and thermal infrared radiative transfer parameterizations used in CCMOB are described by Ramanathan et al. [1983]. These standard parameterizations do not allow for optically active aerosols and must therefore be modified for simulations including smoke or dust. Covey et al. $[1984,1985]$ crudely allowed for smoke by assuming a nonscattering, IR-transparent aerosol. These preliminary studies could be improved upon by implementing a completely generalized solar radiative transfer parameterization that allows for aerosol scattering and absorption in a GCM. This task would not be excessively difficult, but a major problem would arise from the expense (in terms of computer resources) of doing comprehensive, accurate radiative transfer. For example, consider a straightforward, comprehensive scheme based on a delta-Eddington formulation [Joseph and Wiscombe,
1976] for multiple scattering. In this case, a multilayer solar radiative transfer calculation would have to be done for all possible cloud overlap conditions for all spectral intervals (or alternatively all gaseous absorption coefficient probability distribution intervals [Lacis and Hansen, 1974]). Thus at least tens of multilayer calculations per model vertical column would be required for such a comprehensive approach.

A massive level of radiative transfer computation was not feasible for inclusion in our CCM simulations. Moreover, our goal was not to rewrite the complete radiation parameterization of the CCM, but rather to study the atmospheric effects of massive injections of aerosols. Therefore we have adopted an approximate scheme for solar-scattering aerosols that makes use of some existing CCM features and which is not substantially more computationally intensive than the standard CCM solar parameterization. The details of this modified parameterization are given in Appendix A.

Solar radiative transfer is divided into two broadbands as in the standard (CCM): a visible band of $0.25-0.9 \mu \mathrm{m}$ and a near-IR band of $0.9-4.0 \mu \mathrm{m}$. Within the visible band, absorption by ozone (including ultraviolet absorption) is accounted for. Within the near-IR band, water vapor, oxygen, and carbon dioxide absorption are accounted for. Absorptance formulas used are the standard ones in the CCM [Ramanathan et al., 1983]. An approximation for Rayleigh scattering is included. Aerosol scattering and absorption are computed using the delta-Eddington approximation; the aerosol optical properties are discussed in the section describing the model simulations.

Instead of performing multilayer radiative transfer calculations for all possible cloud overlap cases, we have adopted the expedient of defining a single effective cloud layer for the solar radiation computations. The single effective cloud is assumed to occupy a complete single model layer and is derived for each vertical column to represent an average of cloud heights in the column and to provide a reasonable approximation of the total column cloud albedo as viewed from above. The column radiative transfer calculation can thus be divided into two components: a clear-sky component and an overcastsky component. Within the overcast-sky component the calculation is divided into above-cloud and below-cloud partitions, with the single effective cloud layer serving to provide boundary conditions for each partition. After separate calculation, clear- and overcast-sky irradiances and heating rates are weighted according to total cloud cover fraction to determine column-averaged quantities.

\subsection{Thermal Infrared Radsative Transfer}

The standard CCM uses a broadband emissivity and absorptivity parameterization to calculate radiative transfer in the thermal infrared spectral region. Absorptivity and emissivity are used by the model to determine IR irradiances and heating rates [Ramanathan et al., 1983, equations (2)-(3)]. To incorporate the IR properties of aerosols into the CCM, we have developed two modifications of the model's calculation of clear-sky emissivity and absorptivity. (Clouds are assumed in the CCM to behave as blackbodies in the IR and thus are independent of aerosol IR properties.) In one modification the aerosol is treated 


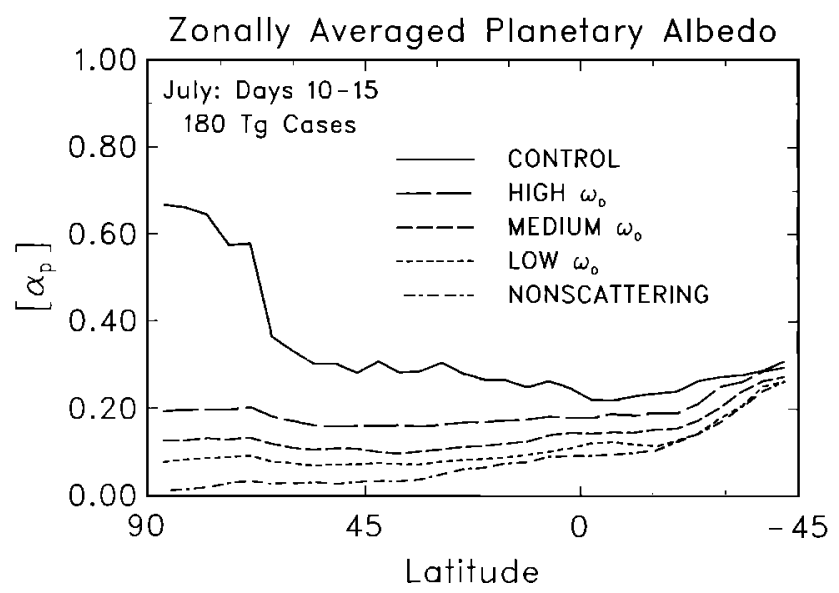

Fig. 1. The zonally averaged planetary albedo averaged over days 10-15 for several July cases. Visible and near-IR single scattering albedos for the cases are high, $\varpi_{o V}=0.75, \varpi_{o N}=0.58$ medium, $\varpi_{o V}=0.60, \varpi_{o N}=0.47 ; \mathrm{low}, \varpi_{o V}=0.45, \varpi_{o N}=$ 0.36 ; and nonscattering, $\varpi_{o V}=\varpi_{o N}=0$. The specific extinction is the same for each case. The control case assumes transparent smoke, i.e., a passive tracer. The total smoke injection is $180 \mathrm{Tg}$; there is no smoke scavenging.

as a "grey" absorber having a constant specific absorption independent of wavelength. From this assumption it follows that clear-sky emissivity and absorptivity may be determined from the product of broadband transmissivity due to gases (computed as described by Ramanathan et al. [1983]) and the wavelength-independent aerosol transmissivity. In the other modification the aerosol specific absorption can be prescribed as a function of four broadband spectral intervals, matching the intervals employed in the standard CCM radiation code to compute gas emissivities and absorptivities, accounting for wavelength dependence of aerosol IR properties to within the wavelength resolution of the CCM radiative transfer algorithm. The details of the modifications are given in Appendix B.

Comparison of results employing the "grey" and "fourband" aerosol IR assumptions allows us to evaluate the sensitivity of the model's predictions to the accuracy of the IR parameterizations. As shown in Appendix B, this sensitivity appears to be quite small; the two parameterizations yield smoke-perturbed average land surface temperatures that differ by $<2 \mathrm{~K}$ (cf. Figure B1 and accompanying discussion).

\section{Simulations}

A baseline smoke scenario is adopted in which 180 teragrams $\left(1 \mathrm{~T}_{\mathrm{g}}=1 \mathrm{~g} \times 10^{12}\right)$ of smoke the baseline smoke amount suggested by the National Research Council (NRC) [1985]) is introduced over NATO and Warsaw Pact territories in July [Thompson, 1985]. Smoke is injected at a constant rate over a period of 2 days, with uniform mixing ratio between the surface and an altitude of $7 \mathrm{~km}$. The model is free to move smoke away from the regions of generation at any time during the injection period and thereafter. The simulation procedure involves running the model under normal conditions for a period of time, then at the initial perturbation time (day 0) a large-scale smoke introduction is started in the specified volume of atmosphere. The action of mesoscale circulations in re- moving smoke from the atmosphere is necessarily ignored in our global circulation model, except for effects implicit in the smoke injection scenario and our model's scavenging parameterizations. Sections 3.1 and 3.2 discuss simulations which do not have smoke scavenging and carry only one tracer field, namely, the smoke volume concentration. These constraints are relaxed in the simulations described in subsequent sections.

\subsection{Solar Scattering: Fixed Optical Properties}

We first examine the model sensitivity to the solar optical properties of smoke. We assume that the solar optical properties of smoke can be prescribed as fixed values for any particular simulation, that there is no IR opacity due to smoke, and that there is no removal of smoke from the atmosphere. Cess et al. [1985] note that a plausible range of smoke single scattering albedo, as used in previous calculations, is $\mathbf{0 . 5}-0.7$. However, smoke produced in urban or industrial fires, because of its high carbon content, can have a single scattering albedo below this range, perhaps as low as $\mathbf{0 . 2 5}$ for smoke from burning petroleum and related materials |Crutzen et al., 1984; NRC, 1985; Penner, 1986]. As "medium-scattering" values, we have chosen the visible single scattering albedo to be $\varpi_{o V}=0.60$, and for the near-IR, $\varpi_{O N}=0.47$. The values are assumed to be valid at wavelengths of 0.51 and 1.55 $\mu \mathrm{m}$ for visible and near-IR, respectively, as derived by Cess et al. [1985] to represent approximately more detailed, spectrally resolved calculations. The ratio of $\varpi_{o V} / \varpi_{o N}$ is taken from the baseline calculation of Cess et al. [1985]. We somewhat arbitrarily define a "high-scattering" case as $\varpi_{o V}=0.75$ and $\varpi_{o N}=0.58$, and a "low-scattering" case as $\varpi_{o V}=0.45$ and $\varpi_{o N}=0.36$. The fixed asymmetry parameters, valid for all three cases, are also taken from Cess et al. $[1985]: \hat{g}_{V}=0.71$ and $\hat{g}_{N}=0.66$.

The specific extinction for all scattering cases is assumed to be $\psi_{e V}=5.50 \mathrm{~m}^{2} \mathrm{~g}^{-1}$ for the visible and $\psi_{e N}=1.45$ $\mathrm{m}^{2} \mathrm{~g}^{-1}$ for the near-IR, as derived from a Mie-scattering calculation for a hypothetical typical smoke composition and size distribution chosen to approximately match the visible smoke absorption used in the $N R C$ [1985] study. The specific absorption in these cases is simply $\psi_{a}=\left(1-\varpi_{0}\right) \psi_{e}$. We define a "nonscattering" case as having the same specific absorption as the medium-scattering case, but a single scattering albedo of zero. For this case, $\varpi_{o V}=\varpi_{o N}=0$, $\psi_{e V}=2.20 \mathrm{~m}^{2} \mathrm{~g}^{-1}$, and $\psi_{e N}=0.77 \mathrm{~m}^{2} \mathrm{~g}^{-1}$. This nonscattering prescription is equivalent to the "purely absorbing" or "nonscattering" smoke assumption used in several previous GCM studies [e.g., Covey et al., 1984, 1985; Thompson, 1985; Malone et al., 1985, 1986].

Figure 1 shows the effect of changes in smoke fixed optical properties on the zonally averaged planetary albedo $\left[\alpha_{p}\right]$ averaged over days $10-15$ of the simulations. A climatologically reasonable pattern of planetary albedo is shown for the "control" case that assumes completely transparent smoke, i.e., in effect, a passive tracer having $\psi_{e}=0$. High albedos appear near the arctic, with a hemispheric mean of about 0.30 . The northern hemisphere planetary albedo drops markedly in all of the smoke cases, including that in the arctic where smoke has spread after 


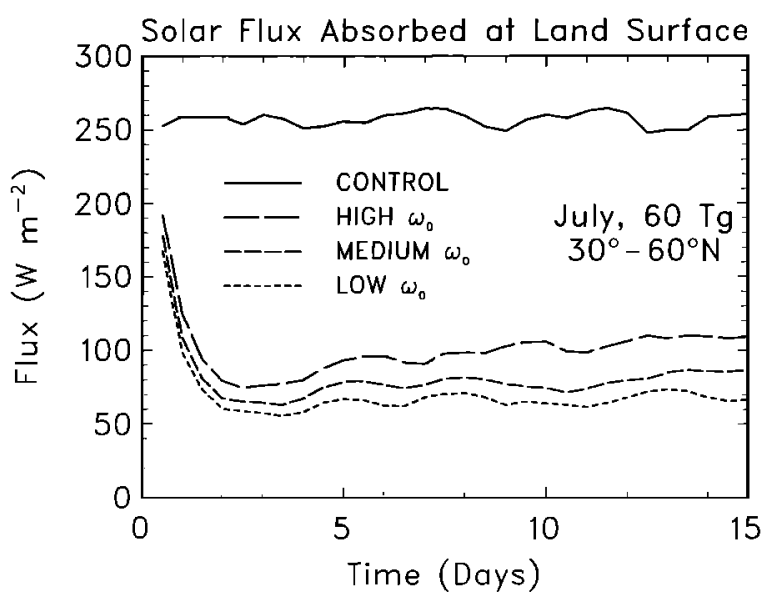

Fig. 2. July time series of the average solar irradiance absorbed at the land surface within the latitude band $30^{\circ}-60^{\circ} \mathrm{N}$ for various scattering cases (see Figure 1). Note that a total smoke injection of $60 \mathrm{Tg}$, instead of $180 \mathrm{Tg}$, is used to allow better differentiation among the cases. There is no smoke scavenging.

less than 10 days. It should be noted that the presence of smoke in the atmosphere acts to suppress the modelcomputed water clouds in the middle to upper troposphere of the model [Thompson, 1985]; this effect contributes to the lowering of planetary albedo in all of the smoke cases. The plausible range of single scattering albedo produces a variation of about 0.1 in planetary albedo, with $\left[\alpha_{p}\right]$ values ranging from 0.1 for low-scattering smoke to 0.2 for highscattering smoke. The nonscattering case produces very low planetary albedos, with $\left[\alpha_{p}\right]$ values ranging from 0.01 to 0.10 in heavily smoke-perturbed areas. Clearly, there is a significant difference between the medium-scattering case and nonscattering case in terms of solar energy absorbed by the earth-atmosphere system; the scattering case absorbs roughly $40 \mathrm{Wm}^{-2}$ less than the nonscattering case over the northern hemisphere for this time period.

Time series of the average solar irradiance absorbed at the land surface within the latitude band $30^{\circ}-60^{\circ} \mathrm{N}$ for the various scattering cases are given in Figure 2 . Note that a total smoke injection of $60 \mathrm{Tg}$, instead of $180 \mathrm{Tg}$, is used to allow better differentiation among the cases $(180 \mathrm{Tg}$ of smoke injection without scavenging tends to block almost all of the solar radiation from the surface regardless of our assumed range of single scattering albedos.) Solar radiation absorbed by the mid-latitude land surface over the first 15 simulation days is reduced from about $260 \mathrm{Wm}^{-2}$ in the control case to a range of 60-110 $\mathrm{Wm}^{-2}$ in the smoke cases. Differences among the smoke scattering assumptions become more pronounced as the smoke spreads, and initially, high optical depths are reduced. (The global spread of smoke and maps of smoke concentration in terms of optical depth are given by Thompson and Giorgi (submitted manuscript, 1987). The solar energy reaching the ground is maximized in the high-scattering case because, for a fixed specific extinction, less solar energy is absorbed in the atmosphere (lower absorption optical depth) in this case. The effect of such scattering-induced differences on land surface temperatures is discussed in section 3.4.

We note that the model used here assumes diurnally averaged solar radiation instead of employing a diurnal cycle of solar forcing. Cess [1985] has shown that more solar energy can reach the surface when a full diurnal cycle is used instead of diurnal averaging since proportionally more energy can reach the surface at small zenith angles than for large zenith angles. Absolute differences in incident surface irradiance between the two calculation methods are largest at intermediate absorption optical depths $\left(\tau_{a} \sim 0.3\right.$ to 1.0$)$ and are about $10-25 \mathrm{Wm}^{-2}$. However, simulations done by Ghan et al. (submitted manuscript, 1987) show only a small surface temperature sensitivity to including the diurnal cycle of insolation.

The effect of solar scattering on the rate of smoke lofting is displayed in Figure 3. Previous GCM simulations which have used the "nonscattering" smoke approximation have found that the solar heating of the atmosphere induced by massive smoke injections acts to perturb the atmospheric circulation in such a fashion as to move the optically absorbing smoke upward much more rapidly than a passive tracer [Thompson, 1985; Malone et al. 1985, 1986]. A comparison of smoke lofting for a $180-\mathrm{Tg}$ injection of nonscattering and medium-scattering smoke is shown in Figure 3. Recall that the two cases employ equivalent specific absorption coefficients for the smoke. The control case, as expected, shows a slow rate of smoke lofting from unperturbed large-scale motions; at the end of 15 days only $6 \%$ of the initially injected transparent smoke is above 10 $\mathrm{km}$ altitude. The optically active smoke cases indicate that nonscattering smoke lofts at a faster rate than scattering smoke. This result follows from Figure 1, which shows that nonscattering smoke absorbs more solar radiation than "equivalent" scattering smoke. The fraction of smoke lofted above $10 \mathrm{~km}$ at the end of 15 days is $33 \%$ for nonscattering and $28 \%$ for scattering, which is not a very large difference.

\subsection{Thermal Infrared: Effect on Surface Temperature Response}

The thermal infrared opacity of smoke is incorporated by making the "grey" absorber approximation which assumes that the specific absorption is independent of wavelength.

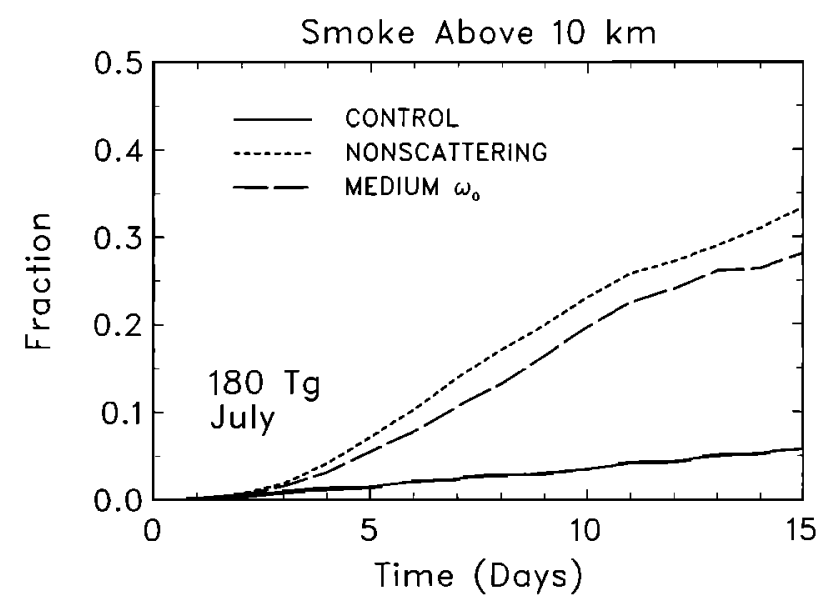

Fig. 3. The global fraction of initially injected smoke that is above $10 \mathrm{~km}$ altitude as a function of time. Initial smoke injection is $180 \mathrm{Tg}$ in July; there is no smoke scavenging. Nonscattering, medium-scattering, and control (transparent smoke) cases are shown. The two optically active smoke cases employ equivalent specific absorption coefficients for the smoke. 


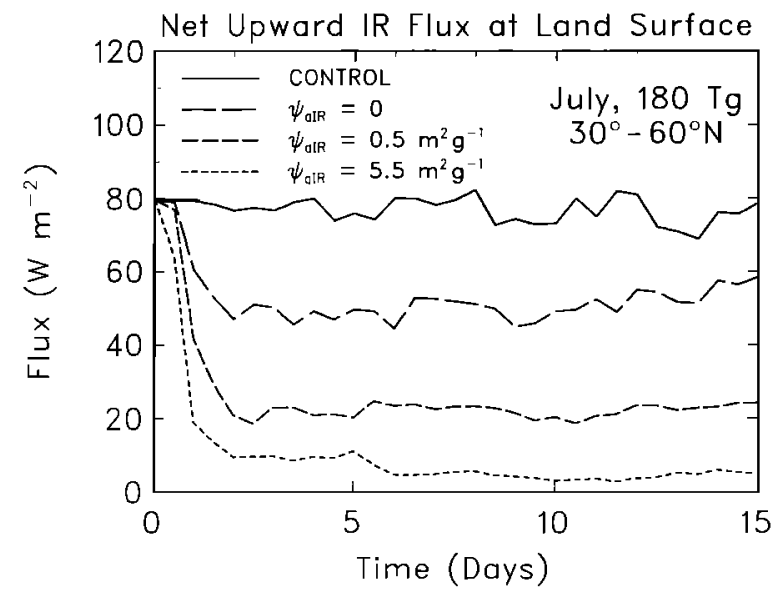

Fig. 4. The average net upward surface thermal IR irradiances as a function of time averaged over the $30^{\circ}-60^{\circ} \mathrm{N}$ land surface for the control case, and for three cases having varying smoke IR specific absorption coefficients $\left(\psi_{a} / R\right)$. The "grey" absorber approximation is used. A smoke injection of $180 \mathrm{Tg}$ is assumed without smoke scavenging.

For the medium-scattering smoke parameters described previously, a nominal smoke IR specific absorption $\left(\psi_{a I R}\right)$ of $0.5 \mathrm{~m}^{2} \mathrm{~g}^{-1}$ is included to replace the previous implicit assumption of IR-transparent smoke. This value of $\psi_{a J R}$ is about 0.1 as large as the specific extinction at visible wavelengths and should be appropriate for typical spherical smoke particles [Ramaswamy and Kiehl, 1985]. When combined with typical values of smoke column loadings the nominal IR specific absorption produces IR optical depths of about 0.2-1.0. In addition, higher IR optical depths are considered in order to assess the effects on surface temperature. Of particular interest is the surface temperature response when both solar and IR opacities are very large. Both the GCM and a one-dimensional radiativeconvective model are employed in this assessment. In the one-dimensional model the IR specific absorption is varied continuously up to $6 \mathrm{~m}^{2} \mathrm{~g}^{-1}$, while holding the column loading fixed. In the GCM a single $\psi_{a I R}$ value of $5.5 \mathrm{~m}^{2}$ $\mathrm{g}^{-1}$ is chosen to define the high-opacity case.

Figure 4 shows the average net upward thermal IR irradiances as a function of time over the mid-latitude land surface for the grey-IR cases. The control case indicates that the July land surface has a net IR loss of about 80 $\mathrm{Wm}^{-2}$ in the absence of smoke. The immediate effects of adding smoke can be deduced most readily by examining the net IR losses at day 1 or 2 , before the atmospheric temperature distribution has had time to respond to altered heating rates. The addition of smoke quickly reduces the net surface IR loss, as (1) downward IR radiation from the smoke reaches the surface in the normal $10-$ to $12-\mu \mathrm{m}$ atmospheric IR window, and (2) the ground surface cools and therefore radiates less IR upward. The two effects are about equal in magnitude, as can be deduced from Figure 4. The net upward IR flux is rapidly decreased to about $50 \mathrm{Wm}^{-2}$ when IR-transparent smoke is added; this effect must therefore be primarily due to a rapid surface temperature decrease. The net upward IR flux is further decreased to about $20 \mathrm{Wm}^{-2}$ when $\psi_{a J R}=0.5 \mathrm{~m}^{2} \mathrm{~g}^{-1}$, and to about 5-10 $\mathrm{Wm}^{-2}$ when $\psi_{a I R}=5.5 \mathrm{~m}^{2} \mathrm{~g}^{-1}$.

The reduction of net surface IR to near zero in the high opacity case is the expected consequence of the tendency to form an isothermal situation in an atmosphere that is opaque at both solar and thermal infrared wavelengths. Thus given $180 \mathrm{Tg}$ of smoke spread over half a hemisphere, our model indicates that smoke IR effects would be at the saturation point for values of $\psi_{a I R}=5.5 \mathrm{~m}^{2} \mathrm{~g}^{-1}$ and larger.

As is implied by the net surface IR curves in Figure 4, surface temperature must increase in typical "greenhouse" fashion with increasing smoke IR opacity. The effect on land surface temperature of the various smoke IR opacity assumptions is shown in Figure 5. The case having a nominal value of $\psi_{a I R}=0.5 \mathrm{~m}^{2} \mathrm{~g}^{-1}$ produces a midlatitude July temperature decrease over days 5-15 that is about $6{ }^{\circ} \mathrm{C}$ smaller in magnitude than that produced by the IR-transparent case. As discussed later, the magnitude of the aerosol IR effect is found to be similar to that in radiative convective models. However, radiative-convective model simulations of similar smoke scenarios produce a $30^{\circ}-40^{\circ} \mathrm{C}$ temperature decrease that would make a $6^{\circ} \mathrm{C}$ variation a relatively small effect. In our GCM, the smoke IR opacity effect can make a substantial relative change in land surface temperature estimates. Increasing the smoke IR specific absorption to the maximum of $5.5 \mathrm{~m}^{2}$ $\mathrm{g}^{-1}$ produces an additional $2^{\circ}-5^{\circ} \mathrm{C}$ surface temperature mitigation, but it does not produce a net surface warming.

Figure 6 shows the result of using the one-dimensional radiative-convective model of Ramaswamy and Kiehl [1985] to examine the land surface temperature effect of smoke IR opacity for a continuous range of "grey" smoke IR specific absorption values and a fixed smoke column loading of 1 $\mathrm{g} \mathrm{m}^{-2}$. The difference in surface temperatures in this model between a control case and a case having smoke but no smoke IR opacity is $42^{\circ} \mathrm{C}$. The surface temperature warming effect increases roughly linearly with IR specific absorption for values less than $1 \mathrm{~m}^{2} \mathrm{~g}^{-1}$. The warming effect appears to asymptote to a value of about $30^{\circ} \mathrm{C}$ for IR specific absorption exceeding $6 \mathrm{~m}^{2} \mathrm{~g}^{-1}$. This behavior is qualitatively consistent with the GCM results described above, although the magnitudes of the one-dimensional model temperature changes are amplified relative to the GCM because of the neglect of oceans.

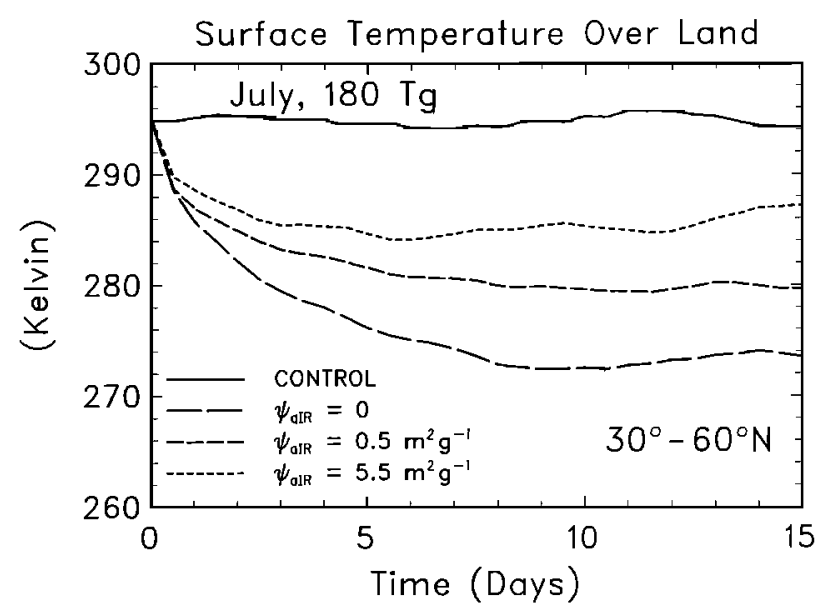

Fig. 5. Time series of July land surface temperature in the $30^{\circ}-60^{\circ} \mathrm{N}$ latitude zone for various assumptions of smoke thermal infrared specific absorption $\left(\psi_{a I R}\right)$. The "grey" absorber approximation is used. There is no smoke Bcavenging. 


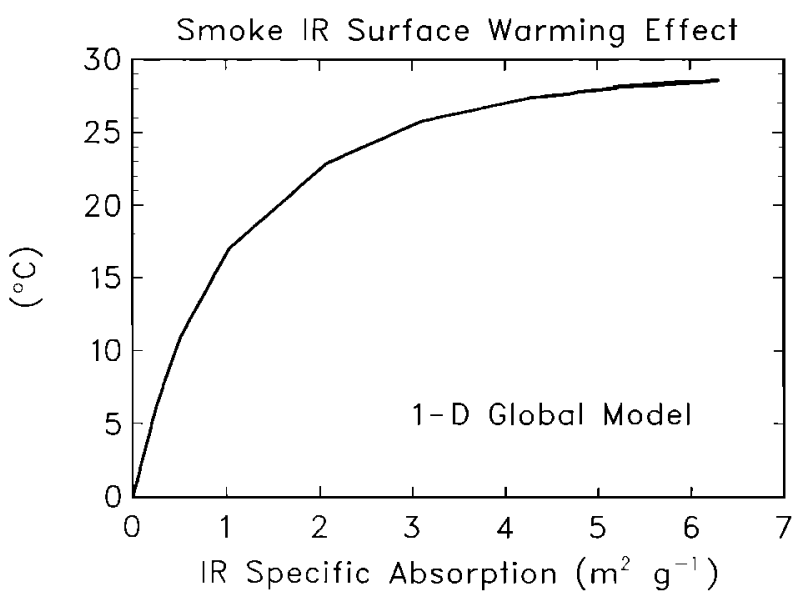

Fig. 6. Land surface temperature moderation (in degrees Celsius) as a function of the thermal infrared specific absorption of smoke. The results are differences between cases with smoke IR opacity minus an IR-transparent case, as derived from a onedimensional radiative-convective model having a smoke loading of $1 \mathrm{~g} \mathrm{~m}^{-2}$ distributed with constant mass mixing ratio over 0-7 $\mathrm{km}$. The difference in surface temperatures in this model between a control case and a case having smoke, but no smoke IR opacity is $42^{\circ} \mathrm{C}$. Thus net surface cooling occurs over the entire range of smoke thermal infrared opacity considered here.

One should note that the large IR optical depths implied in Figure 6 (values up to 6) can be achieved only for very large smoke column loadings or values of specific absorption. Large column loadings in smoke plumes could be present at early times following fires but would not be likely to occur for substantial durations. The possible degree of enhancement of specific absorption due to nonsphericity, porosity, and inhomogeneities |Chyilek et al., 1981] is uncertain, although experiments suggest specific absorption values less than $1 \mathrm{~m}^{2} \mathrm{~g}^{-1}$ [Roessler and Faxvog, 1980].

\subsection{Influence of Vertical Injection Profile on Thermal Infrared Effect}

The moderation of land surface temperature cooling by smoke IR opacity depends on changes in the vertical transfer of thermal IR. Thus it is expected that the vertical concentration profile of the smoke will influence the smoke IR opacity effect. To examine this sensitivity we have performed simulations with the GCM for two different smoke vertical injection profiles. In these simulations the smoke is not only transported but the microphysical removal processes (section 2.1) have been included as well. Given these perturbations to the vertical distribution of smoke, we have attempted to verify the conclusions reached from the GCM studies by performing simulations with a one-dimensional radiative-convective model having several fixed smoke profiles.

Table 1 shows the day 5-15 mean land surface temperature change from control for four GCM cases having injections of $180 \mathrm{Tg}$ of medium scattering smoke. The two smoke injection profiles are $0-7 \mathrm{~km}$ at constant mass mixing ratio (CMR), and $0-10 \mathrm{~km}$ at constant density (CD) concentration. The latter profile corresponds closely to the NRC [1985] baseline case. All else being equal, the higher-altitude $C D$ cases always produce a larger land surface cooling than do the CMR cases. In the $30^{\circ}-50^{\circ} \mathrm{N}$ latitude band the $\mathrm{CD}$ cases are about $3^{\circ} \mathrm{C}$ colder than the CMR cases. A substantial part of this difference probably arises from the slower removal of smoke in the CD cases. At the end of 15 days, $50 \%$ of the initial smoke remains in both CD cases, while only $29 \%$ of the smoke remains in the CMR cases.

In the mid-latitudes the addition of smoke IR opacity produces a land cooling that is about $4^{\circ} \mathrm{C}$ less than the IR-transparent cases. (Note that the value of $4^{\circ} \mathrm{C}$ is smaller than the $6^{\circ} \mathrm{C}$ described in section 3.2 because the removal of smoke included here reduces the total smoke IR opacity.) The IR-opacity effect is insensitive to the injection profile that is chosen, at least in these limited cases. There are two possible general explanations for the insensitivity of the IR-opacity effect to smoke injection profile. First, the GCM smoke undergoes self-lofting and lower-tropospheric removal that can result in smoke profiles after 1-2 weeks of simulation that are similar despite differences in initial injection profiles. Second, there may be compensating factors in the radiative transfer that produce a roughly constant IR-opacity effect over some range of smoke vertical profiles.

Table 2 shows the smoke IR-opacity effect for several three-dimensional and one-dimensional model simulations, given as the change in land surface temperature for cases with smoke IR absorption minus the change with IRtransparent smoke. The one-dimensional model, which is

TABLE 1. July Land Surface Temperature Difference From Control for Two Smoke Vertical Injection Profiles

\begin{tabular}{ccccccc}
\hline & \multicolumn{3}{c}{ Simulation Description } & & \multicolumn{3}{c}{ Temperature Change ${ }^{\circ} \mathrm{C}$} \\
\cline { 7 - 8 } Height, km & Profile & Smoke IR & & $50^{\circ}-70^{\circ} \mathrm{N}$ & $30^{\circ}-50^{\circ} \mathrm{N}$ & $10^{\circ}-30^{\circ} \mathrm{N}$ \\
\hline 7 & CMR & yes & & -12.9 & -10.0 & -3.9 \\
7 & CMR & no & & -17.7 & -13.9 & -5.0 \\
10 & CD & yes & & -14.2 & -13.2 & -8.5 \\
10 & CD & no & -19.9 & -17.2 & -9.8 \\
\hline
\end{tabular}

The difference is equal to perturbed minus control. CMR refers to injection at a constant mass mixing ratio; $C D$ refers to injection at a constant density concentration. Specific absorption for the thermal infrared "grey" approximation is either $0.5 \mathrm{~m}^{2} \mathrm{~g}^{-1}$ (yes) or 0 . (no). Results are averaged over days 5-15. Initial smoke loading is $180 \mathrm{Tg}$ of medium scattering smoke. (This gives an effective visible hemispheric extinction optical depth of 3.88 and absorption optical depth of 1.55.) Smoke scavenging is included. Results for three latitude bands are given. 
TABLE 2. Land Surface Temperature Moderation From Inclusion of the Thermal Infrared Opacity of Smoke Having a Spectrally Uniform Specific Absorption of $0.5 \mathrm{~m}^{2} \mathrm{~g}^{-1}$

\begin{tabular}{lccc}
\hline Vertical Profile & 1-D Clear & 1-D Partly Cloudy & 3-D GCM \\
\hline 0-10 km CD & 7.5 & 5.0 & 4.0 \\
0-7 km CMR & 5.6 & 4.9 & 3.9 \\
3-km scale height & 4.8 & 3.9 & $\ldots$ \\
1-km scale height & 2.9 & 2.8 & $\ldots$ \\
\hline
\end{tabular}

Temperature change is in degrees Celsius. Absorption value is case with smoke IR opacity minus case without. Results in the column labeled "1-D Clear" are from a one-dimensional radiative-convective model with prescribed and fixed vertical smoke profiles, annual mean solar forcing, smoke visible absorption optical depth of 1 , no water clouds, and averaged over days 10-15. The "1-D Partly Cloudy" model has prescribed randomly overlapped water clouds at $3,5.6$, and $10.5 \mathrm{~km}$ at fractional area coverages of $0.31,0.09$, and 0.23 , respectively. The results in the " $3-\mathrm{D} \mathrm{GCM}$ " column are derived from Table 1 for $30^{\circ}-50^{\circ} \mathrm{N}$.

the radiative-convective model described by Ramaswamy and Kiehl [1985], is used for both clear-sky and partly cloudy calculations. The one-dimensional and threedimensional cases are not quantitatively comparable owing to greatly different model resolutions and the treatment of physical processes affecting the smoke (e.g., scavenging and transport). However, the similar qualitative tendencies of both models warrant placing both sets of results in the same table.

In the partly cloudy cases having smoke injection cloud tops in the middle to upper troposphere, the IR-opacity effect is seen to be a roughly constant $5^{\circ} \mathrm{C}$ in the onedimensional model. The ameliorating temperature effect diminishes appreciably only for very low-altitude smoke profiles, e.g., for a 1-km smoke scale height the IR-opacity effect is $2.8^{\circ} \mathrm{C}$. However, in the clear-sky one-dimensional cases the IR-opacity effect increases consistently with increasing height of the smoke cloud top.

The fixed, constant smoke in the partly cloudy onedimensional model eliminates smoke transport and removal as an explanation of the model's insensitivity of the smoke IR effect to vertical injection profile. We thus advance the following explanation for the one-dimensional results in Table 2. First, note that the surface temperature effect of increased smoke IR opacity depends primarily on changes in downward IR flux at the surface if sufficient smoke is present to greatly reduce the solar flux at the surface and produce a strong lower tropospheric inversion. This condition holds for all of the cases in Table 2. Next, note that the downward IR flux at the surface can be roughly divided into an "IR-window" component, originating largely from water clouds or aerosols and an opaque spectral region component, originating from gaseous absorbers in the lower troposphere. The latter component depends primarily on the near-surface temperatures. If solar energy is removed from the surface by smoke absorption well above the surface, then the near-surface temperatures, and by implication the opaque region downward IR fluxes, should be insensitive to the altitude of the smoke cloud. Hence for smoke solar absorption at sufficiently high altitudes, the major factor in changing the downward IR flux at the surface must be the flux in the IR-window region. It is not obvious a priori as to what constitutues "sufficiently high" smoke. But it is very likely that at least the $1-\mathrm{km}$ scale height case in Table 2 violates this assumption.

It is known from simulations, and expected from simple theory, that the temperatures of the smoke-heated region increase as the altitude of the smoke cloud is increased. Since the IR opacity of the smoke in the window region is fixed, the downward window IR from the heated smoke must increase with increasing smoke altitude. This implies a surface temperature effect that increases consistently with increasing smoke cloud altitude. This, in fact, is observed for the clear-sky one-dimensional cases. In the partly cloudy one-dimensional cases the increased IR opacity produced by water clouds in the IR window must fortuitously compensate for the increased downward IR from the hotter high-altitude smoke for cases in which smoke is higher than a $3 \mathrm{~km}$ scale height. The fixed water clouds in the partly cloudy one-dimensional model at $3,5.6$, and $10.5 \mathrm{~km}$ constitute a greater impediment to downward IR from the smoke cloud as smoke altitude increases, the net result being little effective change in the downward window region IR reaching the surface in the CD and CMR cases.

The behavior of the three-dimensional model is similar to that of the one-dimensional partly cloudy model. If the explanation above holds for the three-dimensional model, then the decrease in mid-tropospheric water clouds observed in the smoke-perturbed GCM simulations must not be sufficient to allow the increased downward IR from higher-altitude smoke to reach the surface. Or perhaps other compensating factors are present, such as an increase in low-altitude water clouds. In any case, the warming effect of smoke IR opacity is seen to either increase or remain constant with increasing smoke cloud altitude.

Recent assessments [Scientific Committee on Problems of the Environment (SCOPE), 1987] of the optical properties of nuclear war smoke have suggested that smoke could contain a substanially higher fraction of elemental carbon than assumed in the $N R C$ [1985] smoke baseline case. On the other hand, a plausible estimate of the total smoke mass may not be as high as the $180 \mathrm{Tg}$ assumed in the $N R C$ [1985] baseline. Reasonable estimates of fractional carbon content and smoke mass are still quite uncertain. Therefore we investigate the sensitivity of the model to a scenario 
TABLE 3. July Land Surface Temperature Difference From Control for Two Smoke Scenarios

\begin{tabular}{ccccccc}
\hline & \multicolumn{2}{c}{ Simulation Description } & & \multicolumn{3}{c}{ Temperature Change ${ }^{\circ} \mathrm{C}$} \\
\cline { 1 - 2 } \cline { 5 - 7 } Height, hm & Profile & Smoke IR & & $50^{\circ}-70^{\circ} \mathrm{N}$ & $30^{\circ}-50^{\circ} \mathrm{N}$ & $10^{\circ}-30^{\circ} \mathrm{N}$ \\
\hline 7 & CMR & $180 \mathrm{Tg}$, medium & & -13.9 & -12.9 & -5.9 \\
7 & CMR & $50 \mathrm{Tg}$, sooty & & -16.4 & -14.3 & -6.5 \\
10 & CD & $180 \mathrm{Tg}$, medium & & -14.0 & -15.3 & -10.6 \\
10 & CD & $50 \mathrm{Tg}$, sooty & & -17.8 & -17.0 & -10.1 \\
\hline
\end{tabular}

Difference is equal to perturbed minus control. Here "sooty" refers to smoke that is more highly absorbing of solar radiation than the medium scattering smoke described in section 3.1 (see section 3.3). Results are averaged over days 5-15. Smoke scavenging is not included. Results for three latitude bands are given.

having a higher carbon fraction in a smaller smoke mass. We define a "sooty" smoke having $\varpi_{o V}=\varpi_{o N}=0.2$, $\psi_{\mathrm{eV}}=10.0 \mathrm{~m}^{2} \mathrm{~g}^{-1}$, and $\psi_{\mathrm{eN}}=2.6 \mathrm{~m}^{2} \mathrm{~g}^{-1}$. In terms of solar absorption, the $50 \mathrm{Tg}$ of sooty smoke used in the simulations is the approximate equivalent of $174 \mathrm{Tg}$ of medium-scattering smoke. Thus the $50-\mathrm{Tg}$ sooty and 180-Tg medium-smoke scenarios should produce nearly the same solar absorption.

Table 3 gives the day 5-15 temperature changes from control for sooty- and medium-smoke cases for both CMR and $C D$ vertical injection profiles. (Note that smoke removal is not included in these simulations in order to eliminate a potential source of variation between the sooty- and medium-smoke cases. This also allows for contrasting of the medium-smoke cases in Table 3 with the cases in Table 1 that have removal included.) The $50-\mathrm{Tg}$ sooty-smoke case is seen to produce mid-latitude temperature changes a few degrees larger than the $180-\mathrm{Tg}$ medium-smoke case, despite the equivalent solar absorption potential. This difference is a direct consequence of the smaller smoke IR opacity of the 50 - $\mathrm{Tg}$ versus $180-\mathrm{Tg}$ smoke masses.

\subsection{Solar Scattering: Interactive Optical Properties}

The capability to transport massive amounts of solar scattering smoke in a GCM represents a substantial improvement in simulation realism compared to the initial assumptions of fixed, zonally symmetric, nonscattering smoke. However, the assumption of fixed optical properties is known to be only a very approximate representation of the effects of smoke and other aerosols on atmospheric radiation. Smoke, in reality, undergoes continuous chemical and physical transformations from the time of production to its eventual removal from the atmosphere. The most important of these changes from the standpoint of radiative transfer are size, shape, and composition changes resulting from (1) chemical reactions and (2) coagulation or agglomeration. The time evolution of smoke chemical composition is a potentially important problem, since it affects the hygroscopic nature of the particles (hence the potential for nucleation scavenging) as well as the optical properties. The evolution of smoke composition should be pursued in the future, but it is beyond the scope of this study.

Time- and space-dependent changes in smoke particle size and shape can, in principle, be used to calculate smoke optical properties. However prohibitively time-consuming Mie-scattering calculations, the typical way of computing aerosol optical properties, are not suited for direct inclusion in a GCM. Therefore we have parameterized the results of detailed Mie-scattering computations and have performed preliminary simulations to test the potential of such parameterizations. The microphysical scavenging processes that are approximated in our simulations (see section 2.1) provide $r_{g}$ that vary with time and geographical position. This particle size information is then used to estimate the local optical properties of the smoke: specific extinction $\psi_{e}$, specific absorption $\psi_{a}$, and asymmetry parameter $\hat{g}$. The single scattering albedo is easily diagnosed from $\psi_{e}$ and $\psi_{a}$. The smoke optical properties are derived assuming that smoke particles have a density of $1 \mathrm{~g} \mathrm{~cm}^{-3}$ and a lognormal distribution having $\ln \left(\sigma_{g}\right)=0.3$. Parameterizations for three different smoke compositions are used, corresponding approximately to the light, medium, and dark smokes discussed in section 3.1. The complex indices of refraction of the parameterized smokes at solar wavelength are: light, $m=1.5-0.05 i$; medium, $m=1.5-0.10 r$; and dark, $m=1.5-0.45 \imath$. The optical properties $\psi_{e}, \psi_{a}$, and $\hat{g}$ as a function of $r_{g}$, for both the solar visible $(\lambda=0.51 \mu \mathrm{m})$ and the near-IR $(\lambda=1.55 \mu \mathrm{m})$, as derived from the Miescattering calculations, are approximated by fifth-degree polynomials valid for particle radii of $0.05 \mu \mathrm{m}<r_{g}<1.0$ $\mu \mathrm{m}$. The polynomial curve fits are accurate to better than $10 \%$ throughout almost all of their range of validity.

Our preliminary interactive optical properties scheme makes the following simplifying assumptions:

1. Smoke composition is fixed.

2. Smoke particles can be represented optically as spheres.

3. Smoke optical properties do not depend on changes in the standard deviation $\sigma_{g}$ of the lognormal distribution. (This is not true, but Mie-scattering tests show that the optical properties do depend more on $r_{g}$ than on $\sigma_{g}$.)

4. The thermal-IR optical properties (i.e., specific absorption) are fixed and computed using the "grey" absorber' approximation; only the solar optical properties are parameterized.

Figure 7 shows a typical evolution of smoke particle size, as taken from a simulation in which $180 \mathrm{Tg}$ of smoke is injected in the model that includes microphysical removal processes. Thompson and Giorgi (submitted manuscript, 1987) discuss such results in more detail; the purpose of Figure 7 is to demonstrate the range of $r_{g}$ typically 


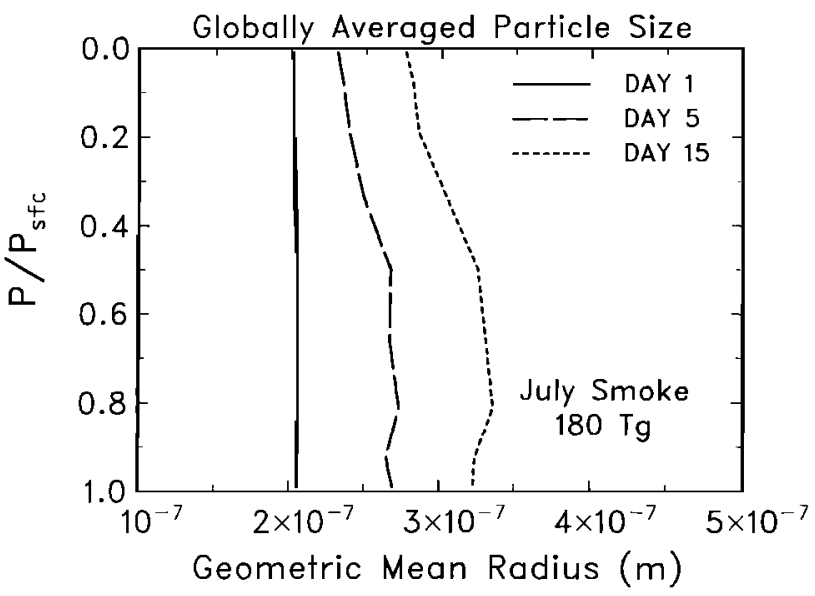

Fig. 7. The global massweighted value of geometric mean smoke radius $\left(r_{g}\right)$ as a function of height for three times during a July simulation of a $180-\mathrm{Tg}$ injection of smoke. The model includes microphysical removal processes, as discussed by Thompson and Giorgi (submitted paper, 1987). Smoke radii increase due to coagulation, and they increase most rapidly where the smoke is initially most concentrated $(0-7 \mathrm{~km})$. The vertical axis $\left(P / P_{\circ f c}\right)$ is pressure normalized by surface pressure.

encountered in our simulations. Coagulation is responsible for an increase in $r_{g}$ from a uniform initial value of 0.2 to $0.28-0.33 \mu \mathrm{m}$ by day 15 . Rapid coagulation creates larger values of $r_{g}$ where the smoke cloud is most dense during the first few days of the simulation (i.e., $0-7 \mathrm{~km}$ ).

The sensitivity of smoke optical properties and smoke layer transmittance to smoke particle size, as determined by our interactive optical properties parameterization, are illustrated in Table 4. For our "medium" composition smoke the visible specific extinction decreases from 8.24 $\mathrm{m}^{2} \mathrm{~g}^{-1}$ to $5.25 \mathrm{~m}^{2} \mathrm{~g}^{-1}$ for an increase of $r_{g}$ from 0.2 to $0.35 \mu \mathrm{m}$. The resulting decrease in smoke opacity is partially compensated by increased fractional absorption, as indicated by the decrease in single scattering albedo from 0.681 to 0.612 over the same size range. The resulting smoke layer transmittances for two different smoke mass loadings are also given in Table 4 . The differences in layer transmittance over the full range of smoke particle size considered in Table 4 could create differences in July absorbed solar irradiance at the surface of $5-30 \mathrm{Wm}^{-2}$, depending on smoke column loading and cloud cover.

We have performed simulations using the interactive optical properties parameterization and have compared one of these cases to a simulation using the fixed optical properties prescription. Both cases assume an injection of $180 \mathrm{Tg}$ of medium composition smoke. The fixed properties case is prescribed by simply fixing the value of $r_{g}$ used in computing optical properties. A constant $r_{g}=0.2 \mu \mathrm{m}$ was chosen to match the initial smoke particle radius used in these simulations. (To be precise, $r_{g}$ does vary in the fixed properties case; only the value used in the optical properties polynomials is kept constant.) The results of both simulations are quite similar, e.g., the zonally averaged northern hemisphere mid-latitude land surface temperatures differ by only $1^{\circ}-2^{\circ} \mathrm{C}$ between the two simulations. This rather small difference is explained by (1) the large optical depths at early times that act to minimize the differences in solar energy reaching the surface and (2) the moderate change in smoke radii from 0.20 to $0.28 \mu \mathrm{m}$ in the upper troposphere (see Figure 7) where the bulk of the smoke is concentrated after 1015 days. One should not be mislead, however, by the relatively small differences between these particular cases. Plausible variations in smoke particle size can produce substantial changes in the transfer of solar radiation, as has been demonstrated in one-dimensional radiative transfer calculations [Ramaswamy and Kiehl, 1985].

Figure 8 displays the potential influence of plausible variations in smoke solar optical properties on land surface temperature. The cases shown use the interactive optical properties parameterization, scavenging, and the "grey" aerosol-IR approximation with $\psi_{a I R}=0.5 \mathrm{~m}^{2} \mathrm{~g}^{-1}$. Temperature decreases over the $30^{\circ}-50^{\circ} \mathrm{N}$ latitude zone reach their maximum between days 5 and 10, after which an apparent slow recovery begins. The range of smoke composition from "dark" to "light" produces a $4^{\circ}-6^{\circ} \mathrm{C}$ difference in average land surface temperature over days 5-15 of the simulations. This amounts to about a $\pm 20-30 \%$ variation in surface temperature cooling for these cases. Thus at least for these July $180-\mathrm{Tg}$ simulations, plausible variations in smoke composition can make a substantial difference in surface temperature estimates.

\section{Discussion}

\subsection{Model Intercomparisons}

The temperature changes given in Table 1 are not as large as the changes predicted for comparable smoke scenarios in previous climate simulations of nuclear war smoke. In particular, Thompson [1985] used a version of the

TABLE 4. Specific Extinction, Single Scattering Albedo, Asymmetry Parameter, and Transmittance in the Visible Spectral Interval For Smoke Layers Having Different Geometric Mean Radii and Column Density

\begin{tabular}{lccccc}
\hline & \multirow{2}{*}{$\psi_{c V}}$, \\
$r_{g}, \mu \mathrm{m}$ & $\left(\mathrm{m}^{2} \mathrm{~g}^{-1}\right)$ & $\varpi_{o V}$ & $\hat{g}_{V}$ & $0.1 \mathrm{~g} \mathrm{~m}^{-2}$ & $0.5 \mathrm{~g} \mathrm{~m}^{-2}$ \\
\hline 0.20 & 8.24 & 0.681 & 0.758 & 0.514 & 0.046 \\
0.25 & 7.46 & 0.672 & 0.810 & 0.553 & 0.061 \\
0.30 & 6.38 & 0.647 & 0.839 & 0.592 & 0.082 \\
0.35 & 5.25 & 0.612 & 0.852 & 0.631 & 0.108 \\
\hline
\end{tabular}

Here $\lambda=0.51 \mu \mathrm{m}$ is the spectral interval used. Two different column densities, $0.1 \mathrm{~g} \mathrm{~m}^{-2}$ and $0.5 \mathrm{~g} \mathrm{~m}^{-2}$, are used. The optical properties of smoke are taken from the parameterization discussed in section 3.4; the complex index of refraction is $m=1.5-0.10$. 


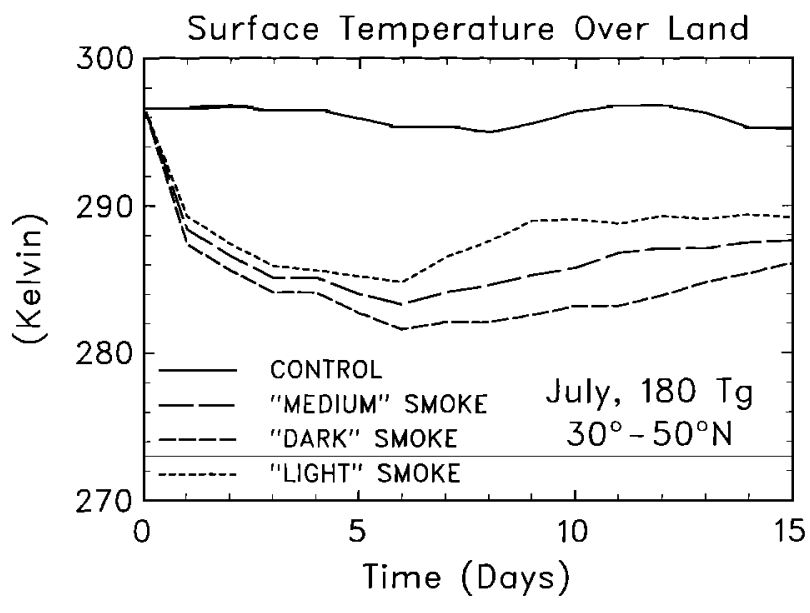

Fig. 8. Time series of land surface temperature in the $30^{\circ}-50^{\circ} \mathrm{N}$ latitude zone for cases having different smoke compositions. All cases shown use the interactive optical properties parameterization, scavenging and the "grey" aerosol-IR approximation with $\psi_{a J R}=0.5 \mathrm{~m}^{2} \mathrm{~g}^{-1}$. "Light," "medium," and "dark" smoke refer to complex indices of refraction of $m=1.5-0.05 i, m=1.5-0.10 i$, and $m=1.5-0.452$, respectively, at solar wavelengths. Scaveng. ing is included.

GCM used here that did not have smoke-scavenging, solarscattering, or smoke IR opacity. Thompson [1985] found a $19^{\circ} \mathrm{C}$ land surface temperature drop in the $30^{\circ}-50^{\circ} \mathrm{N}$ band for the CMR smoke scenario and temperature-averaging conditions described here in Table 1 . The current $30^{\circ}-$ $50^{\circ} \mathrm{N}$ temperature drop of $10^{\circ} \mathrm{C}$ is the result of first adding smoke removal to go from $19^{\circ} \mathrm{C}$ to $13.9^{\circ} \mathrm{C}$ (cf. Table 1) and then adding smoke IR opacity to get to $10^{\circ} \mathrm{C}$. The alternative path is to first add IR opacity to go from $19^{\circ} \mathrm{C}$ to $12.9^{\circ} \mathrm{C}$ (cf. Table 3) and then to add smoke removal to get to $10^{\circ} \mathrm{C}$. The "path-dependent" effect arises from the dependence of the smoke IR opacity on smoke amount.

Although it is difficult to comprehensively intercompare models as complicated as GCMs, at the suggestion of $S$. J. Ghan of the Lawrence Livermore National Laboratory (LLNL) we have contrived a smoke scenario and simple analysis procedure suitable for use in both the GCM described here and the model used by Ghan et al. (submitted manuscript, 1987). The scenario is $150 \mathrm{Tg}$ of smoke injected in July, with a uniform mass mixing ratio up to the tropopause; solar specific extinction of $5 \mathrm{~m}^{2} \mathrm{~g}^{-1}$ and specific absorption of $2 \mathrm{~m}^{2} \mathrm{~g}^{-1}$ for both visible and near-IR bands; specific absorption in the thermal IR of 0.5 $\mathrm{m}^{2} \mathrm{~g}^{-1}$; no dust; no smoke scavenging; no diurnal cycle; neutral-stability boundary layer, and surface fluxes. The comparison is done for land temperatures averaged over $30^{\circ}-70^{\circ} \mathrm{N}$ for days $5-15$ of the simulations. The results for both surface interface (ground surface) and surface air (lowest model atmospheric) temperature are given in Table 5. The intermodel differences are about as small as could be expected given the different model resolutions and initial conditions. Indeed, the difference between NCAR and LLNL models is smaller than the difference between surface and surface air temperatures. The substantial difference in surface temperatures that arises from the strong near-surface inversion suggests that in the future researchers precisely specify the type of surface temperature they report. All of the surface temperatures in this work are surface interface temperatures, except as noted in Table 5 .

\subsection{Conclusion}

Global atmospheric models of massive nuclear war aerosol injections have made substantial advances over the past few years in the comprehensiveness of their simulations. But while important enhancements have been made, substantial improvements remain to be incorporated. Covey et al. [1985, p. 5626] described global modeling areas where improvements were thought necessary for more realistic simulations. The list included radiative transfer, planetary boundary layer and surface processes, subgrid-scale vertical convection, and tracer transport and removal.

This study has attempted to improve upon some of the earlier aerosol radiative transfer approximations employed in GCMs. Previous assumptions of nonscattering, thermal IR-transparent smoke have been replaced by more comprehensive radiative transfer parameterizations. A plausible range of smoke-scattering assumptions is found to produce a significant, but not overwhelming, variation in surface temperature response to solar obscuration. The inclusion of IR absorption by smoke aerosols is found to be a potentially significant factor in mitigating land surface temperature decreases. The sensitivity of atmospheric effects to both these factors suggests that future GCM modeling should include them for improved realism. The ability to interactively compute the optical properties of aerosols will be important in future GCM simulations of optically important aerosols that undergo chemical and microphysical evolution in the atmosphere, particularly when climatic changes over time scales of months to years are simulated. Our preliminary attempts in this study indicate that simulations with interactive optical properties can be done economically if detailed scattering calculations (or empirical data) can be parameterized for inclusion in GCMs.

The present effort to improve the GCM calculation of radiative transfer through dense aerosols still has shortcomings, however. One problem is that the approximate solar radiative transfer scheme we have developed is not completely general and will not be accurate for multiple layers of very dense, highly scattering aerosols. Moreover, the "single effective cloud" approximation we employ for water clouds should be replaced by a multilayer parameterization.

TABLE 5. Change in Day 5-15 Land Surface and Land Surface Air Temperatures Over the Latitude Band $30^{\circ}-70^{\circ} \mathrm{N}$ for Two General Circulation Models Having Nearly Equivalent Smoke Scenarios

\begin{tabular}{lcc}
\hline & \multicolumn{2}{c}{ Temperature Change ${ }^{\circ} \mathrm{C}$} \\
\hline & Surface Air & Surface \\
\hline NCAR & -11.9 & -15.6 \\
LLNL & -13.7 & -18.7
\end{tabular}

Details of the scenario are given in section 4.1. "NCAR" refers to the model version used in the current study; "LLNL" refers to the model version used by Ghan et al. (submitted manuscript, 1987). (LLNL results from S. J. Ghan, personal communication, 1987.) 
The dependence of smoke optical properties on particle size, shape, and composition at both solar and thermal infrared wavelengths is an area that also needs much more work. The assumption of spherical particles necessitated by Mie theory is almost certainly invalid for typical smoke aerosols. However, the degree of error introduced by the assumption of sphericity is poorly quantified at present. Future simulations employing interactive smoke optical properties should attempt to better account for the nonspherical, agglomerated morphology of smoke.

More straightforward problems are our assumptions of diurnally averaged insolation, zero heat capacity land surface, and a neutral-stability formulation for surface and lower tropospheric heat and moisture fluxes. The inclusion of a diurnal cycle of insolation will require some model improvements in the representation of surface processes and subgrid-scale vertical mixing. In the simulations described in this study, we have made no improvements in these areas since the detailed description of near-surface problems given by Covey et al. [1985]. Some recent results, however, can be cited regarding the potential magnitude of temperature errors resulting from incomplete near-surface processes. Ghan et al. (submitted manuscript, 1987) find that including a stability-dependent vertical mixing prescription acts to enhance mid-latitude land surface cooling by from 20 to $40 \%$, with the larger enhancement occurring at early times. In a higher-resolution GCM of the British Meteorological Office, J. Mitchell [SCOPE 1987 finds a $20 \%$ increase in land surface cooling when stability-dependent vertical mixing is included, but a $20 \%$ decrease in land surface cooling when a zero heat capacity surface is replaced by a four-layer heat-transporting soil model. These effects are time-dependent, however; e.g., the effect of adding soil heat conduction is largest during the first few days of the simulations.

The transport and removal of optically active aerosols has also been improved in our simulations. Thompson and Giorgi (submitted manuscript, 1987) describe these enhancements in more detail than considered here. The lifetime of climatically important aerosols will play a large role in determining the chronic, long-term effects of massive aerosol injections and the amount of aerosol transported out of source regions. At present, transport and removal parameterizations have doubtful validity on both very short and very long time scales. Short time scale problems are dominated by cloud and mesoscale processes that cannot be explicitly simulated in GCMs. On the other hand, long time scale simulations will require transport and removal processes that produce accurate stratospheric aerosol lifetimes.

General circulation models with the capability to simulate the injection, transport and removal of optically active aerosols should prove valuable for atmospheric studies beyond the problem of nuclear war aerosols that has prompted the current development of such models. "Dust" has long been studied as an aerosol of climatic significance. Comprehensive simulations of the transport and removal of volcanic aerosol or Saharan dust should provide further insights into the climatic impact of these events, as well as helping to verify the models. Dust is also used as a paleoclimatic "proxy" indicator of climatic change. GCMs with aerosol transport may be useful in examining notions of paleoatmospheric circulation change and surface changes in the regions of potential dust genesis. Smoke too is now known to have a potential climatic significance. Arctic haze, an aerosol originating from industrial combustion, might be studied using models generally similar to the one used in this study, but having a higher resolution. Furthermore, enough natural and anthropogenic smoke is now created worldwide to pose potentially an interesting global climatic question in itself. It seems clear that atmospheric circulation models capable of comprehensive aerosol simulations will be useful tools in the study of many atmospheric problems.

\section{Appendix A: Solar Radiative Transfer}

Solar radiative transfer is divided into two broadbands, as in the standard Community Climate Model: a visible band of $0.25-0.9 \mu \mathrm{m}(64.7 \%$ of insolation) and a near-infrared band of $0.9-4.0 \mu \mathrm{m}$ (35.3\% of insolation). Within the visible band, absorption by ozone is accounted for (including ultraviolet absorption). Within the near-IR band, water vapor, oxygen, and carbon dioxide absorption are accounted for. Absorptance formulas used are the standard ones in the CCM [Ramanathan et al., 1983]. The distribution of ozone is specified as a function of time of year; oxygen and carbon dioxide assume the present constant atmospheric mixing ratio; and the water vapor distribution, of course, depends on the model's interactive hydrologic cycle. The treatment of Rayleigh scattering and aerosols is described later. Solar radiation boundary conditions consist of prescribed diurnally averaged insolation, using a solar constant of $1370 \mathrm{Wm}^{-2}$; the prescribed surface albedo depends on surface type and spectral interval.

Cloud cover is predicted in five of nine model layers. Thus numerous potential vertical cloud overlap conditions can occur. Instead of performing multilayer radiative transfer calculations for all possible cloud overlap cases, we have adopted the expedient of defining a single effective cloud layer for the solar radiation computations. The single effective cloud is assumed to occupy a complete single model layer and is derived for each vertical column to represent an average of cloud heights in the column and to provide a reasonable approximation of the total column cloud albedo, as viewed from above. The column radiative transfer calculation can thus be divided into two components: a clear-sky component and overcastsky component. Within the overcast-sky component the calculation is divided into above-cloud and below-cloud partitions, with the single effective cloud layer serving to provide boundary conditions for each partition. After separate calculation, clear- and overcast-sky irradiances and heating rates are weighted according to total cloud cover fraction to determine column-averaged quantities.

\section{A.1. Single Effective Cloud Properties}

The total single effective cloud cover fraction $C_{S}$ is derived on the assumption of random overlap of the clouds in each model layer. Thus

$$
C_{S}=1-\prod_{k=k_{C b}}^{k_{C t}}\left(1-C_{k}\right)
$$


where $C_{k}$ is the cloud amount in model layer $k$, and $k_{C b}, k_{C t}$ are the lowest and highest model layers that can contain clouds, respectively. In the standard nine-layer CCM version we use, $k_{C b}=3$ and $k_{C t}=7$.

The albedo of the single effective cloud layer is derived for both visible and near-IR solar bands. For each band two types of albedo are defined: one for direct beam downward solar flux $\left(\alpha_{S}^{\prime}\right)$ and one for diffuse upward solar flux $\left(\alpha_{S}^{*}\right)$. In either case the effective albedo is computed by weighting the cloud albedos of the individual layers. The individual layer weights are proportional to two factors: the cloud amount fraction in the layer and a measure of the obscuration of the cloud layer to the relevant incident solar flux. This latter estimated "visibility factor" is proportional to the total clear-sky fraction lying between the cloud layer of interest and the source of the incident flux and to the clear-sky transmissivity of the atmosphere between the incident irradiance source and the cloud.

The effective single-layer cloud albedo to downward direct flux is

$$
a_{S}^{\prime}=\sum_{k=k_{C b}}^{k_{C t}} W_{k}^{\prime} C_{k} \alpha_{k}^{\prime} / \sum_{k=k_{C b}}^{k_{C t}} W_{k}^{\prime} C_{k}
$$

where $W_{k_{C \mathrm{t}}}^{\prime}=1$, and $W_{k}^{\prime}=W_{k+1}^{\prime}\left(1-C_{k+1}\right) T_{k+1}^{\prime}$ for $k_{C b} \leq k \leq k_{C t}-1 ; T_{k}^{\prime}$ is the clear-sky atmospheric transmissivity of layer $k$ to downward direct flux; and $\alpha_{k}^{\prime}$ is the albedo of clouds in layer $k$ to downward direct flux (see discussion of cloud albedo in section A.4).

The effective single-layer cloud albedo to upward diffuse flux is

$$
\alpha_{S}^{*}=\sum_{k=k_{C b}}^{k_{C t}} W_{k}^{*} C_{k} \alpha_{k}^{*} / \sum_{k=k_{C b}}^{k_{C t}} W_{k}^{*} C_{k}
$$

where $W_{k_{C b}}^{*}=1$, and $W_{k}^{*}=W_{k-1}^{*}\left(1-C_{k-1}\right) \tau_{k-1}^{*}$ for $k_{C b}+1 \leq k \leq k_{C t} ; T_{k}^{*}$ is the clear-sky atmospheric transmissivity of layer $k$ to upward diffuse flux, and $\alpha_{k}^{*}$ is the albedo of clouds in layer $k$ to downward diffuse flux.

The transmissivities of the single effective cloud to downward direct and upward diffuse solar radiation, $T_{S}^{\prime}$ and $T_{S}^{*}$, respectively, are defined as in (A2) and (A3), except that cloud layer transmissivity replaces albedo.

The height of the single effective cloud is important, since it determines the partition of above-cloud and below-cloud regions that are treated differently in our overcast-sky solar radiative transfer scheme. The model layer containing the single effective cloud is defined by weighting the layer values (or equivalently, the heights) of the individual layers containing clouds. We assume that for solar calculations at least, high clouds are more important than lower clouds in the same column in the sense that high clouds can obscure the influence of lower clouds on solar radiative transfer. Thus the layer-weighting factor is derived for solar irradiance incident from above. The layer number of the single effective cloud is then

$$
k_{S}=\sum_{k=k_{C b}}^{k_{C t}} W_{k} C_{k} k / \sum_{k=k_{C b}}^{k_{C t}} W_{k} C_{k}
$$

where $W_{k_{C t}}=1$, and $W_{k}=W_{k+1}\left(1-C_{k+1}\right)$ for $k_{C b} \leq$ $k \leq k_{C t}-1$.
These derivations for a single effective cloud are, of course, only approximate, since it is impossible to define a single cloud layer that can account for all of the effects of multilayer clouds. For example, a single cloud will disturb the relative distribution of upper and lower tropospheric solar heating. This effect will be most pronounced when comparing smoke above and below the cloud. But over many time and space samples these errors should average out to some extent.

\section{A.2. Multilayer Radiative Transfer}

The solar radiative transfer scheme we use to calculate broad band irradiances and heating rates requires the reflectivity $R$ and transmissivity $T$ of each model layer, the calculation of which is described in sections below. The radiative transfer scheme for clear sky above the surface is shown schematically in Figure A1. In essence, the incoming solar irradiance at the top of the atmosphere is followed downward through the atmosphere to the surface. As the downward directed "beam" penetrates the atmosphere it is depleted by back-scattering (computed using layer $R$ values) and absorption (implied from layer $1-T-R$ values). Scattering produces incremental upward directed diffuse irradiances from each layer. The downward directed irradiance itself includes both direct and downward diffuse (forward scattered) components. The upward diffuse fluxes generated in each layer, including surface reflection, are then summed upward (allowing for atmospheric transmissivity) to get the upward diffuse irradiance. In a sufficiently scattering atmosphere this upward directed irradiance can itself give rise to more interlayer multiple reflections. Thus an additional set of secondary downward and upward diffuse irradiances are derived and added to the primary irradiances to approximately account for clear-sky interlayer reflections.

There are two major potential sources of inaccuracy in our simplified multilayer scheme: (1) incomplete accounting of interlayer reflections, and (2) inaccurate treatment of layer transmissivities of diffuse irradiances. The latter source of error arises because layer transmissivities due to gaseous absorbers (e.g., $\mathrm{H}_{2} \mathrm{O}$ ) are based on nonexponential broadband formulas |Ramanathan et al., 1983]. Thus the previous absorber path of the irradiance incident on a layer partially determines the layer transmissivity. This effect is accurately accounted for in the first downward and upward passes through the atmosphere, but for simplicity, additional multiple reflection passes assume a diffuse transmissivity that is independent of the previous path.

The accuracy of the interlayer scattering component of the parameterization has been evaluated by comparison with a general method of known accuracy. We have performed tests with a homogeneous atmosphere where irradiances were calculated with (1) a single-layer deltaEddington approximation, and (2) the parameterization described above with the homogeneous atmosphere divided into five sublayers. The results of some of these tests are given in Tables A1 and A2. In general, our simplified multilayer parameterization is reasonably accurate under the following conditions: (1) scattering by aerosols having a low single scattering albedo for any optical depth, or 


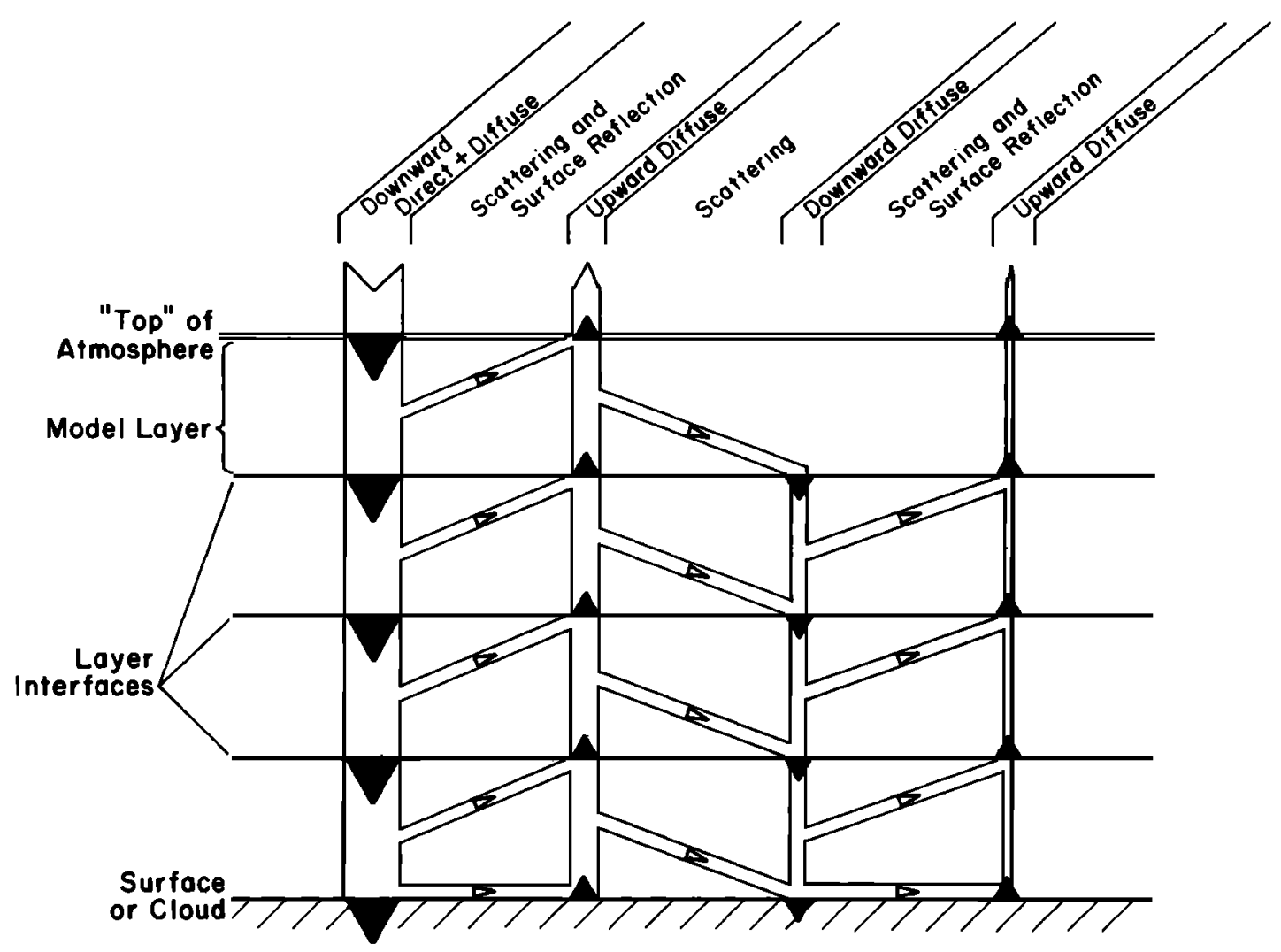

Fig. A1. Schematic of the solar multilayer radiative transfer scheme for clear sky above a cloud or terrestrial surface. Horizontal lines represent model vertical layer boundaries (in this case, a four-layer model). Vertical bars denote direct and diffuse solar irradiances. Black arrowheads at layer interfaces indicate the location and direction (up or down) of defined irradiances. To read this illustration, start at the upper left corner, which indicates the downward direct irradiance at the top of the atmosphere, and scan each vertical bar, following the arrowheads, and moving from left to right. As the downward direct "beam" penetrates the atmosphere, scattering occurs which produces incremental upward directed diffuse irradiances from each layer. These incremental irradiances are indicated by the upward slanting bars to the right of the downward beam. The downward beam itself includes both direct and downward diffuse irradiances. The upward diffuse fluxes, including surface reflection, are then summed to get the upward diffuse irradiance (the upward directed vertical bar second from the left). This procedure is repeated once more to allow approximately for secondary atmosphere/surface multiple reflections, as denoted by the last two vertical bars on the right.

(2) scattering for any single scattering albedo for small optical depths. That is, our scheme is reasonably accurate in terms of radiative fluxes if multiple reflections between atmospheric model layers are not too large relative to transmission. Tables A1 and A2 show that this condition is met for smoke at any optical depth, but it is not met for more highly scattering aerosols (dust, water clouds) unless the total optical depth is less than about 1 .

In the overcast-sky case the procedure is (1) to independently compute the nondimensional, normalized irradiances (i.e., assuming a unit incoming irradiance) for above-cloud and below-cloud partitions (described later); (2) to use the normalized downward irradiance at the top of the single effective cloud layer to provide a boundary condition for the below-cloud irradiances, thus allowing them to be scaled to match the above-cloud irradiances; (3) based on the below-cloud calculation, to define the effective cloud/surface albedo for downward directed irradiances at the cloud top; (4) to use the effective cloud/surface albedo as a boundary condition to compute the dimensional above- cloud irradiances; and (5) to use the downward dimensional irradiance at the cloud top to compute the dimensional irradiances below the cloud.

The normalized irradiances in the above-cloud partition are computed in a manner identical to that for clear sky described previously (see Figure A1), except that the surface albedo in the clear-sky computation is replaced by the underlying effective cloud/surface albedo in the overcast-sky case. The normalized below-cloud irradiances are computed in a manner similar to the clear-sky case, but with the following exceptions: (1) the below-cloud atmosphere is assumed to be nonscattering, and (2) two cloud/surface reflection passes are accounted for instead of the one atmosphere/surface reflection pass allowed for in the clear-sky case. The first exception is a simplification based on the assumption that additional Rayleigh or aerosol scattering below the already highly scattering cloud will have little effect on layer heating rates or solar irradiance at surface. The second exception accounts for the larger multiple reflections expected in overcast-sky situations. 
TABLE A1. Comparison of Transmissivities From the Multilayer Radiative Transfer Scheme With a Single Layer Delta-Eddington Reference Calculation

\begin{tabular}{rcccccr}
\hline$\tau$ & $\varpi_{0}$ & M.L. & Ref. & \% Err. & Diff. & W m $^{-2}$ \\
\hline 0.20 & 0.6000 & 0.818 & 0.818 & -0.1 & -0.000 & -0.2 \\
1.00 & 0.6000 & 0.370 & 0.376 & -1.8 & -0.007 & -2.3 \\
5.00 & 0.6000 & 0.008 & 0.011 & -29.3 & -0.003 & -1.1 \\
0.20 & 0.9200 & 0.910 & 0.910 & -0.1 & -0.001 & -0.2 \\
1.00 & 0.9200 & 0.643 & 0.653 & -1.5 & -0.010 & -3.4 \\
5.00 & 0.9200 & 0.146 & 0.181 & -19.2 & -0.035 & -11.8 \\
0.20 & 0.9800 & 0.928 & 0.929 & -0.1 & -0.001 & -0.2 \\
1.00 & 0.9800 & 0.715 & 0.725 & -1.4 & -0.010 & -3.5 \\
5.00 & 0.9800 & 0.271 & 0.331 & -18.3 & -0.061 & -20.6 \\
0.20 & 0.9999 & 0.934 & 0.935 & -0.1 & -0.000 & -0.2 \\
1.00 & 0.9999 & 0.741 & 0.751 & -1.4 & -0.010 & -3.5 \\
5.00 & 0.9999 & 0.335 & 0.412 & -18.6 & -0.077 & -26.0 \\
\hline
\end{tabular}

For each optical depth $\tau$ and single Bcattering albedo $\varpi_{0}$, the multilayer (M. L.) scheme as tested here divides the total optical depth into five equal layers and approximates the radiative transfer, as discussed in section A2. The comparison with the reference (Ref.) result is given as percentage error in total transmissivity for direct and diffuse radiation, difference, and difference in terms of energy flux below the layer, assuming an incoming direct beam irradiance of $340 \mathrm{Wm}^{-2}$. The four values of single scattering albedo are chosen to be generally representative of smoke $(0.6)$, soil dust (0.92), glassy dust (0.98), and water clouds (0.9999).

Finally, the clear-sky and overcast-sky irradiances and derived heating rates are combined into partly cloudy column averages using the total single effective cloud cover fraction $C_{S}$ as the weighting factor.

\section{A.9. Rayleigh Scattering}

The visible broadband reflectivity of atmospheric layers due to Rayleigh scattering is derived by first determining the Rayleigh optical depth $\tau_{R}$, then employing a curve fit to determine the layer reflectivity $R_{R}$ in terms of $\tau_{R}$ and the cosine of the solar zenith angle $\mu$. The visible band-integrated $(0.35-0.9 \mu \mathrm{m})$ Rayleigh optical depth is given by

$$
\tau_{R}=K \Delta p
$$

where $K=\left(K^{*} R T_{0}\right) /\left(g p_{0}\right)=1.4457 \times 10^{-6} \mathrm{~Pa}^{-1}$. Here $K^{*}$ is a scattering coefficient equal to $1.7156 \times 10^{-5} \mathrm{~m}^{-1}$, $R$ is the gas constant for air, $g$ is gravitational acceleration, $T_{\circ}$ is a reference temperature $(288 \mathrm{~K}), p_{o}$ is a reference pressure $\left(10^{5} \mathrm{~Pa}\right)$, and $\Delta p$ is the atmospheric layer pressure thickness. ( $K^{*}$ is derived by spectrally weighting and averaging Rayleigh monochromatic optical depths.)

We define $\tau^{*} \equiv \tau_{R} / \mu$ and

$$
x \equiv \frac{2 \tau^{*}-\tau_{b}^{*}-\tau_{a}^{*}}{\tau_{b}^{*}-\tau_{a}^{*}}
$$

where $\tau_{a}^{*}=0.001$ and $\tau_{b}^{*}=1.500$. The reflectivity of an atmospheric layer is then given by a polynomial curve fit based on detailed multiple scattering calculations:

$$
\begin{aligned}
R_{R}=a_{0} & +a_{1} x+a_{2} x^{2}+a_{3} x^{3}+a_{4} x^{4} \\
& +a_{5} x^{5}+a_{6} x^{6}+a_{7} x^{7}+a_{8} x^{8}
\end{aligned}
$$

The polynomial coefficients are

\begin{tabular}{|c|c|c|c|c|c|c|}
\hline$\tau$ & $\varpi_{0}$ & M.L. & Ref. & \% Err. & DifT. & $W m^{-2}$ \\
\hline 0.20 & 0.6000 & 0.035 & 0.034 & 1.2 & 0.000 & 0.1 \\
\hline 1.00 & 0.6000 & 0.090 & 0.086 & 4.3 & 0.004 & 1.3 \\
\hline 5.00 & 0.6000 & 0.101 & 0.098 & 2.9 & 0.003 & 1.0 \\
\hline 0.20 & 0.9200 & 0.059 & 0.058 & 1.0 & 0.001 & 0.2 \\
\hline 1.00 & 0.9200 & 0.210 & 0.202 & 3.9 & 0.008 & 2.7 \\
\hline 5.00 & 0.9200 & 0.363 & 0.350 & 3.8 & 0.013 & 4.5 \\
\hline 0.20 & 0.9800 & 0.064 & 0.063 & 0.9 & 0.001 & 0.2 \\
\hline 1.00 & 0.9800 & 0.245 & 0.236 & 3.8 & 0.009 & 3.0 \\
\hline 5.00 & 0.9800 & 0.522 & 0.507 & 3.0 & 0.015 & 5.1 \\
\hline 0.20 & 0.9999 & 0.066 & 0.065 & 0.9 & 0.001 & 0.2 \\
\hline 1.00 & 0.9999 & 0.258 & 0.249 & 3.7 & 0.009 & 3.2 \\
\hline 5.00 & 0.9999 & 0.601 & 0.587 & 2.3 & 0.013 & 4.5 \\
\hline
\end{tabular}

$$
\begin{aligned}
& a_{0}=1.9724273 \times 10^{-1} \\
& a_{2}=-3.7827285 \times 10^{-2} \\
& a_{4}=-5.8154239 \times 10^{-3} \\
& a_{6}=-8.7333261 \times 10^{-4} \\
& a_{8}=-6.7299686 \times 10^{-4}
\end{aligned}
$$

TABLE A2. The Same as Table A1, Except the Comparison is for Layer Reflectivity 
$\tau_{a}^{*}$ and $\tau_{b}^{*}$ are the limits of validity of the polynomial above. $R_{R}$, as defined previously, includes the approximate effect of the absorption of ultraviolet radiation in the stratosphere. This correction reduces $R_{R}$ because ultraviolet radiation that would normally be strongly scattered is depleted above the denser lower atmosphere where Rayleigh scattering becomes appreciable.

\section{A.4. Individual Cloud Layer Properties}

The determination of the albedo and transmissivity of the single effective cloud layer requires the albedos and tranmissivities of all of the model-predicted cloud layers. These individual cloud layers are assuned to be plane-parallel with fixed, prescribed optical properties unaffected by other aerosols. Given the optical depth $\tau_{C}$, asymmetry parameter $\hat{g}_{C}$, and single scattering albedo $\varpi_{o C}$ of each cloud layer, a delta-Eddington calculation is done to compute the cloud layer albedo and transmissivity to both direct and diffuse solar irradiances $\left(\alpha^{\prime}\right.$ and $\alpha^{*} ; T_{C}^{\prime}$ and $\tau_{C}^{*}$, respectively). In practice, the diffuse properties are computed as direct beam properties using an effective diffuse cosine of the solar zenith angle of $\mu=0.5$. The layer albedos and transmissivities are weighted as described in section A.1 above to get values for the single effective cloud.

Counting model layers upward from the surface, the cloud layer optical depths are prescribed as a function of height as follows: layer $3(1-2 \mathrm{~km}), \tau_{C}=7$; layers $4-5$ $(2-7 \mathrm{~km}), \tau_{C}=12 ;$ layer $6(7-10 \mathrm{~km}), \tau_{C}=7$; layer 7 $(10-15 \mathrm{~km}), \tau_{C}=2$. These values are used for both visible and near-IR spectral intervals.

Here $\hat{g}_{C}$ and $\varpi_{O C}$ are assumed to be independent of height. In the visible spectral interval, $\hat{g}_{C}=\mathbf{0 . 8 4 0}$ and $\varpi_{O C}=0.99999$. In the near-IR spectral interval, $\hat{g}_{C}=0.800$ and $\varpi_{O C}=0.985$ [e.g., Ramaswamy and Kiehl, $1985]$.

\section{A.5. Aerosols}

The contributions to the reflectivity and transmissivity of atmospheric layers due to aerosols for both direct and diffuse solar irradiance $\left(R_{A}^{\prime}\right.$ and $R_{A}^{*} ; \tau_{A}^{\prime}$ and $\tau_{A}^{*}$, respectively) are, as for water clouds, computed on-line using the delta-Eddington approximation. The optical properties of smoke are either prescribed fixed values or are parameterized as a function of particle radius based on Mie-scattering calculations, as described in the main text. The optical properties of dust are always prescribed fixed values. Smoke and dust optical depths, asymmetry parameters, and single scattering albedos are combined as described by Cess [1985] to create a single "effective" aerosol.

\section{A.6. Layer Total Transmissivity and Reflectivity}

The multilayer solar radiative transfer parameterization described in section $A .2$ requires the total reflectivity $\mathcal{R}$ and transmissivity $T$ for each model layer. Thus the various contributions to layer reflectivity and transmissivity arising from gaseous absorbers, Rayleigh scattering, and aerosols must be appropriately combined. In the following, the superscript prime and asterisk, indicating direct and diffuse irradiance, respectively, have been dropped, since the discussion applies equally to both types of irradiance.

In the visible spectral interval the effects of Rayleigh scattering and aerosol scattering/absorption are combined, based on a test of the aerosol opacity. If the aerosol opacity is sufficiently small, then it is ignored. On the other hand, if the aerosol opacity is sufficiently large, then the Rayleigh reflectivity is ignored. Specifically, if the aerosol transmissivity $\tau_{A}>0.9957$ in any model layer, then the combined Rayleigh/aerosol reflectivity is $R_{R A}=R_{R}$, and the combined Rayleigh/aerosol transmissivity is $\tau_{R A}=1-$ $R_{R}$. Otherwise, if the aerosol transmissivity $\tau_{A} \leq 0.9957$, then the combined Rayleigh/aerosol reflectivity is $R_{R A}=$ $R_{A}$, and the combined Rayleigh/aerosol transmissivity is $\tau_{R A}=\tau_{A}$. The value of $\tau_{A}=0.9957$ was chosen somewhat arbitrarily as roughly representing an aerosolgenerated solar heating rate of order $(0.3) \mathrm{K}$ day $^{-1}$, which is approximately one tenth of the maximum solar heating rates found in the model when aerosols are not included.

Ozone absorbs in both visible and ultraviolet spectral intervals. In our parameterization the visible spectral interval implicitly includes the ultraviolet spectral interval. Therefore the total ozone transmissivity is incorporated to get the total visible transmissivity:

$$
T=T_{R A} T_{O_{3}}
$$

where $T_{O}$ is the ozone transmissivity. The total reflectivity is assumed to be unaltered by ozone absorption, thus

$$
R=R_{R A}
$$

In the near-IR spectral interval the only sources of scattering are aerosols, thus in the near-IR band

$$
R=R_{A}
$$

The near-IR transmissivity is, however, decreased by gaseous absorbers. Thus

$$
\tau=\tau_{A}\left(1-A_{\mathrm{H}_{2} O}-A_{O_{2}}-A_{C O_{2}}\right)
$$

where the $A_{x}$ terms represent the near-IR absorptivities of water vapor, oxygen and carbon dioxide.

\section{A.7. Comparison With Standard Parameterization}

The modified solar parameterization has been compared to the standard CCM parameterization by performing a 120-day simulation without aerosols. It was found that the modified parameterization, despite its numerous differences, matches the standard scheme quite well. Quantities of primary importance to the global energy balance and atmospheric dynamics agree closely with the original scheme; e.g., the difference in the planetary albedo is less than 0.02 at all latitudes and the difference in stratospheric heating rates is less than $0.1^{\circ} \mathrm{C} d a y^{-1}$. Time-averaged temperatures and winds generated with the two different parameterizations indicated no differences that could be considered obviously significant in a 4-month simulation. Thus no supplementary "tuning" (e.g., of cloud properties) 
was needed to reach adequate agreement with the original model.

\section{Appendix B. Infrared Radiative Transfer}

\section{B.1. Radiative Transfer Algorthms}

To incorporate the thermal infrared properties of smoke and dust aerosols into the CCM, we modify the model's calculation of clear-sky broadband emissivity and absorptivity. (The model assumes that clouds are blackbodies in the thermal IR, so modification of cloud thermal IR properties is not necessary.) Emissivity $\varepsilon$ and absorptivity $a$ are defined by

$$
\varepsilon=\left(\sigma T^{4}\right)^{-1} \int\left(1-T_{\omega}\right) B_{\omega} d \omega
$$

and

$$
a=\left(4 \sigma T^{3}\right)^{-1} \int\left(1-\tau_{\omega}\right) \frac{d B_{\omega}}{d T} d \omega
$$

where $\sigma$ is the Stefan-Boltzman constant, $T$ is temperature, $\omega$ is frequency, $B_{\omega}$ is the Planck blackbody monochromatic irradiance, $\tau_{\omega}$ is the monochromatic transmissivity of the total combination of gases and aerosols, and the integrals extend over the entire atmospherically significant thermal IR range, 4-500 $\mu \mathrm{m}$. Absorptivity and emissivity are used by the model to determine thermal IR fluxes and heating rates Ramanathan et al., 1983, equations (2)-(3)].

In the "grey aerosol" approximation, gas and aerosol broadband transmissivities are assumed to be multiplicative, i.e.,

$$
\varepsilon=1-\tau_{0} \tau_{d}+\left(\varepsilon_{\mathrm{H}_{2} \mathrm{O}}+\varepsilon_{\mathrm{O}_{3}}+\varepsilon_{\mathrm{CO}_{2}} \bar{\tau}_{\mathrm{H}_{2} \mathrm{O}}^{(3)}\right) \tau_{0} \tau_{d}
$$

and similarly for $a$. In (B3) $\tau_{b}$ and $\tau_{d}$ denote smoke and dust transmissivities, respectively, and the expression in parenthesis is broadband gas transmissivity computed by the standard CCMO radiation code. Here $\varepsilon_{\mathrm{H}_{2} \mathrm{O}}, \varepsilon_{\mathrm{CO}_{2}}$, $\varepsilon_{\mathrm{O}_{3}}$ are emissivities of single gas species in the absence of other species, and $\bar{T}_{\mathrm{H}_{2} \mathrm{O}}^{(3)}$ is the transmissivity of water vapor in the $12-$ to $16-\mu \mathrm{m}$ interval, which overlaps with $\mathrm{CO}_{2}$ emissivity.

In the "four-band" approximation a simplification of the procedure of Ramaswamy and Kiehl [1985] is employed to compute $\varepsilon$ and $a$. Monochromatic transmissivities are assumed to be multiplicative, i.e.,

$$
\tau_{\omega}=T_{\mathrm{H}_{2}} \mathrm{O} T_{\mathrm{CO}_{2}} T_{\mathrm{O}_{3}} T_{d} \tau_{d}
$$

where $T_{x}$ refers to the monochromatic transmissivity due to species $x$ alone. With this assumption, both $\varepsilon$ and $a$ can be rewritten in the following form:

$$
\begin{aligned}
\varepsilon=\varepsilon_{\mathrm{H}_{2} O} O & +\varepsilon_{A: H_{2} O}+\varepsilon_{O_{3}} \tau_{0}^{(2)} \tau_{d}^{(2)} \\
& +\varepsilon_{\mathrm{CO}_{2}} \bar{\tau}^{(3)} \bar{\tau}_{d}^{(3)} \tau_{\mathrm{H}_{2} \mathrm{O}}^{(3)}
\end{aligned}
$$

where

$$
\varepsilon_{A: H_{2} O}=\left(\sigma T^{4}\right)^{-1} \int\left(1-T_{a} \tau_{d}\right) T_{H_{2}} O B_{\omega} d \omega
$$

and $\bar{\tau}_{x}^{(i)}$ denotes the average transmissivity of species $x$ in frequency band $i$. We divide the thermal IR regime into four bands: 4-8 $\mu \mathrm{m}(i=1), 8-12 \mu \mathrm{m}(i=2), 12-16 \mu \mathrm{m}$ $(i=3)$, and $16-500 \mu \mathrm{m}(i=4)$.

In (B5), $\bar{\tau}_{b}{ }^{(2)} \bar{\tau}_{d}^{(2)}$ is an average of $\tau_{b} \tau_{d}$ over the 8to $12-\mu \mathrm{m}$ wavelength interval, weighted by the Planck function and by $\left(1-\tau_{\mathrm{O}_{3}}\right)$. In deriving this term it is assumed (consistent with the CCM radiation scheme) that ozone absorption is negligible outside the 8- to $12-\mu \mathrm{m}$ band and that water vapor absorption is negligible inside this band. (That is, water vapor continuum absorption is ignored in the derivation of (B7) that follows.) Likewise, $\bar{\tau}_{a}^{(3)} \bar{\tau}_{d}^{(3)} \bar{\tau}_{\mathrm{H}_{2} \mathrm{O}}^{(3)}$ is the analogous average of $\tau_{d} \tau_{d} \tau_{\mathrm{H}_{2} \mathrm{O}}$ over the 12- to $16-\mu \mathrm{m}$ interval, weighted by the Planck function and by $\left(1-T_{\mathrm{CO}_{2}}\right)$. This last weighting factor is assumed to be negligible outside the $12-$ to $16-\mu \mathrm{m}$ band. The term $\varepsilon_{A: H_{2} O}$ is computed in a manner consistent with the other terms on the right-hand side of (B5):

$$
\varepsilon_{A: H_{2} O}=\sum_{i=1}^{4}\left(1-\bar{\tau}_{d}^{(i)} \tau_{d}^{(i)}\right) \tilde{\tau}_{H_{2} O}^{(i)} p^{(i)}
$$

where $p^{(i)}$ is the fraction of Planck blackbody irradiance contained in frequency band $\imath$ (parameterized as a linear function of temperature).

Equations (B5) and (B7) allow one to compute $\varepsilon$ given the average transmissivities in the four bands $\bar{\tau}_{x}^{(i)}$. (The standard version of the CCM radiation code already computes $\varepsilon_{\mathrm{H}_{2} \mathrm{O}}, \varepsilon_{\mathrm{O}_{3}}$ and $\varepsilon_{\mathrm{CO}_{2}}$; these computations are not altered by the aerosol modifications, and thus our modified code reverts to the original CCM thermal IR treatment in the absence of smoke and dust.) The band-averaged smoke and dust aerosol transmissivities between any two vertical levels are assumed to follow Beer's Law with the parameterization of zenith angle dependence suggested by Rodgers and Walshaw [1966]:

$$
\bar{\tau}_{x}^{(i)}=\exp \left[-1.66 c_{x}^{(i)} u_{x}\right], \quad x=s \text { or } x=d
$$

where $u_{x}$ is the column amount (mass per unit area) of $x$ between the levels and $c_{x}^{(i)}$ is the band-average of the appropriate monochromatic absorption coefficient [e.g., Ramaswamy and Kiehl, 1985, Table 3]. Water vapor transmissivities are given by

$$
\bar{\tau}_{\mathrm{H}_{2} \mathrm{O}}^{(i)}=A_{2} \exp \left(B_{i} u_{H_{2} \mathrm{O}}\right)
$$

where $u_{H_{2} O} \mathrm{O}$ is the pressure-weighted column amount of water vapor. For the 12- to $16-\mu \mathrm{m}$ interval, $A_{i}$ and $B_{i}$ are already determined by the unmodified CCM radiation code; for the other wavelength intervals, $A_{i}$ and $B_{i}$ are obtained from a fit to the results of Rodgers' and Walshaw's model.

Absorptivity $a$ is determined by formulations analogous to (B5)-(B7).

It is instructive to consider the "four-band" parameterization of aerosol thermal IR effects in the limiting case in which $\tau_{s}$ and $\tau_{d}$ are independent of $\omega$. Making the assumption

$$
\bar{\tau}_{x}^{(i)}=\tau_{x}, \quad x=s \text { or } x=d, \quad \imath=1,2,3,4
$$




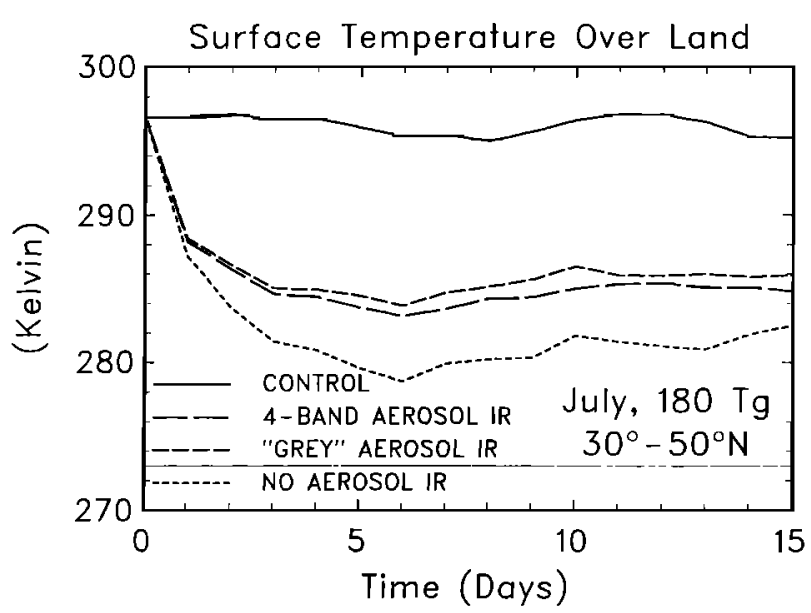

Fig. B1. Time series of land surface temperature in the $30^{\circ}$ $50^{\circ} \mathrm{N}$ latitude zone for cases having different parameterization of thermal IR absorption by aerosols. All cases shown use the interactive optical properties parameterization for July injections of $180 \mathrm{Tg}$ of medium composition smoke, having a complex index of refraction of $m=1.5-0.10 i$ at solar wavelengths. The IR. specific absorption coefficient is $\psi_{a J R}=0.5 \mathrm{~m}^{2} \mathrm{~g}^{-1}$ in the "grey" absorber case. The specific absorption coefficients in the fourband case are $\psi_{a / R^{1}}=0.70 \mathrm{~m}^{2} \mathrm{~g}^{-1}$ for $4-8 \mu \mathrm{m}, \psi_{a} / R^{2}=0.45$ $\mathrm{m}^{2} \mathrm{~g}^{-1}$ for $8-12 \mu \mathrm{m}, \psi_{\mathrm{a} l R^{3}}=0.28 \mathrm{~m}^{2} \mathrm{~g}^{-1}$ for $12-16 \mu \mathrm{m}$, and $\psi_{a / R^{4}}=0.15 \mathrm{~m}^{2} \mathrm{~g}^{-1}$ for $16-500 \mu \mathrm{m}$. Scavenging is included.

in (B5) one finds that the "grey aerosol" parameterization (equation (B3)) is recovered, provided that

$$
\varepsilon_{A: H_{2} O}=\left(1-T_{8} \tau_{d}\right)\left(1-\varepsilon_{H_{2} O}\right)
$$

From (B1) and (B6) it is evident that (B11) will be satisfied if $\varepsilon_{\mathrm{H}_{2} \mathrm{O}}$ and $\varepsilon_{\mathrm{A} . \mathrm{H}_{2} \mathrm{O}}$ are computed with consistent water vapor transmissivities and wavelength resolution. This condition is not precisely met in our formulation, because $\varepsilon_{H_{2}} \mathrm{O}$ is computed (by the original CCM radiation scheme) with the parameterization of Sasamori [1972], while $\varepsilon_{\mathrm{A} \mathrm{H}_{2} \mathrm{O}}$, computed according to (B7), is based on the results of Rodgers and Walshaw [1966]. In practice, the discrepancy is minor; aerosol tests in which (B10) is assumed show no important difference in heating rates from the "grey aerosol" tests in which (B3) is imposed directly in the model.

\section{B.2. Comparsson of "Grey" and "Four-Band" Results}

The thermal IR opacity of smoke was shown to be potentially significant in reducing the magnitude of land surface temperature changes created by the obscuration of solar radiation at the surface (section 3.2 ). Since this IR effect appears to be important, we have examined the sensitivity of the model to changes in the IR parameterization by comparing model results obtained with the "grey" and "four-band" aerosol IR parameterizations. Using data from Ramaswamy and Kiehl [1985], the IR specific absorption coefficients used in the four-band approximation are $\psi_{a I R^{1}}=0.70 \mathrm{~m}^{2} \mathrm{~g}^{-1}$ for $4-8 \mu \mathrm{m}, \psi_{a I R^{2}}=0.45 \mathrm{~m}^{2}$ $\mathrm{g}^{-1}$ for 8-12 $\mu \mathrm{m}, \psi_{\mathrm{a} I R^{3}}=0.28 \mathrm{~m}^{2} \mathrm{~g}^{-1}$ for $12-16 \mu \mathrm{m}$, and $\psi_{a I R^{*}}=0.15 \mathrm{~m}^{2} \mathrm{~g}^{-1}$ for $16-500 \mu \mathrm{m}$. The specific absorption coefficient is a constant $\psi_{a I R}=0.5 \mathrm{~m}^{2} \mathrm{~g}^{-1}$ in the comparable "grey" absorber parameterization.
The simulations discussed in this section include dust as well as smoke. The effects of the dust are unimportant for the qualitative conclusions reached here concerning the parameterization of smoke IR opacity. However, the dust does quantitatively influence the simulations; thus in the interest of an accurate description of the model, we describe the dust scenario employed. (Thompson and Giorgi (submitted manuscript, 1987) describe the interactive effects of dust "solar shielding" on smoke transport and the effects of smoke heating on dust transport.) Following the tropospheric and stratospheric baseline dust estimates of the $N R C$ [1985] study, $40 \mathrm{Tg}$ of submicron dust is injected between 7 and $15 \mathrm{~km}$ altitude over the same areas as smoke. The dust is characterized by the following solar optical properties: $\varpi_{o V}=0.98, \varpi_{o N}=0.98, \psi_{\mathrm{eV}}=2.7 \mathrm{~m}^{2} \mathrm{~g}^{-1}$, $\psi_{\mathrm{eN}}=2.2 \mathrm{~m}^{2} \mathrm{~g}^{-1}, \hat{g}_{V}=0.70$, and $\hat{g}_{N}=0.69$. (These properties are derived by assuming the dust to be of glassy, basaltic composition having complex indices of refraction of $m=1.53-0.001 i$ at $\lambda=0.51 \mu \mathrm{m}$ and $m=1.53-0.0022 i$ at $\lambda=1.55 \mu \mathrm{m}$.) The "grey" IR approximation is used for dust, with a specific absorption of $0.1 \mathrm{~m}^{2} \mathrm{~g}^{-1}$.

Time series of land surface temperature in the $30^{\circ}-50^{\circ} \mathrm{N}$ latitude zone for cases having different parameterizations of thermal IR absorption by aerosols are given in Figure B1. All cases shown use the interactive optical properties parameterization for July injections of $180 \mathrm{Tg}$ of medium composition smoke having a complex index of refraction of $m=1.5-0.102$ at solar wavelengths. The principal feature of interest in Figure $\mathrm{Bl}$ is the close correspondence between the four-band and grey IR cases. The four-band case shown in Figure $\mathrm{B} 1$ is only slightly colder $\left(1^{\circ}-2^{\circ} \mathrm{C}\right)$ than the grey absorber case. From this result it appears that the effect of IR absorption by smoke can be reasonably approximated by a "grey" absorber having a spectrally invariant $\psi_{a I R}$ equal to the value in the 10- to $12-\mu \mathrm{m}$ atmospheric window region. Indeed, the slight difference between the two cases could be due in part to the difference in $\psi_{a I R}$ of $0.5 \mathrm{~m}^{2}$ $\mathrm{g}^{-1}$ for the grey case and $\psi_{a I R^{2}}=0.45 \mathrm{~m}^{2} \mathrm{~g}^{-1}$ for the 8to $12-\mu \mathrm{m}$ interval in the four-band case.

Acknowledgments. The authors are grateful to J. T. Kiehl for helpful discussions on the thermal infrared radiative transfer perameterization and for comments on the manuscript, and to $S$. $H$. Schneider, F. Giorgi and R. C. Malone for comments on the manuscript. This work was sponsored by the Defense Nuclesr Agency under task code RB RB/OPERATIONS and work unit 00074/GLOBAL CLIMATE MODELING (Work Unit Manager: David Auton), by the Lawrence Livermore National Laboratory (Department of Energy DE-AI03-84SF 12208), and by the $\mathrm{Na}$ tional Science Foundation (ATM-8504807). The National Center for Atmospheric Research is sponsored by the National Science Foundation.

\section{REFERENCES}

Aleksandrov, V. V., and G. L. Stenchikov, On the modelling of the climatic consequences of the nuclear war, The Proceeding on Applied Mathematics, The Computing Centre of the USSR Academy of Sciences, Moscow, 1983.

Cess, R. D., Nuclear war: Illustrative effects of atmospheric smoke and dust upon solar radiation, Clim. Change, 7, 237-251, 1985.

Cess, R. D., G. L. Potter, S. J. Ghan, and W. L. Gates, The climatic effects of large injections of atmospheric smoke and dust: $A$ study of climate feedback mechanisms with one- and three-dimensional climate models, J. Geophys. Res., 90, 12,937$12,950,1985$.

Chervin, R. M., Interannual variability and seasonal climate predictability, J. Atmos. Sci., 49, 233-251, 1986. 
Chýlek, P., V. Ramaswamy, R. Cheng, and R. G. Pinnick, Optical properties and mass concentration of carbonaceous smokes, Appl. Opt., 20, 2980-2985, 1981.

Covey C., S. H. Schneider, and S. L. Thompson, Global atmospheric effects of massive smoke injections from a nuclear war: Results from general circulation model simulations, Nature, 908 , 21-25, 1984 .

Covey, C., S. L. Thompson, and S. H. Schneider, "Nuclear Winter": A diagnosis of atmospheric general circulation model simulations, J. Geophys. Res., 90, 5615-5628, 1985.

Crutzen, P. J. and J. W. Birks, The atmosphere after a nuclear war: Twilight at noon, Ambro, 11, 114-125, 1982.

Crutzen, P. J., I. E. Galbally, and C. Brühl, Atmospheric effects from post-nuclear fires, Clim. Change, 6, 323-364, 1984.

Ghan, S. J., M. C. MacCracken, and J. J. Walton, Climatic response to large summertime injections of smoke into the atmosphere: Changes in precipitation and the Hadley circulation, Tech. Note UCRL-92324, Lawrence Livermore Natl. Lab., Livermore, Calif., 1985.

Joseph, J. H., and W. J. Wiscombe, The delta-Eddington approximation for radiative flux transfer, J. Atmos. Sct., 99, 2452$2459,1976$.

Lacis, A. A. and J. E. Hansen, A parameterization for the absorption of solar radiation in the Earth's at mosphere, J. Atmos. Sci., $91,118-133,1974$.

MacCracken, M. C., and J. J. Walton, The effects of interactive transport and scavenging of smoke on the calculated temperature change resulting from large amounts of smoke, Tech. Note UCRL-91446, Lawrence Livermore Natl. Lab., Livermore, Calif., 1984.

Malone, R. C., L. H. Auer, G. A. Glatzmaier, M. C. Wood, and $O$. B. Toon, Influence of solar heating and precipitation scavenging on the simulated lifetime of post-nuclear war smoke, Scrence, 290, 317-319, 1985.

Malone, R. C., L. H. Auer, G. A. Glatzmaier, M. C. Wood, and $O$. B. Toon, Nuclear winter: Three-dimensional simulations including interactive transport, scavenging, and solar heating of smoke, J. Geophys. Res., 91, 1039-1053, 1986.

National Research Council, The Effects on the Atmosphere of a Major Nuclear Exchange, 193pp., National Academy Press, Washington, D.C., 1985.

Penner, J. E., Progress in developing the smoke source term for "nuclear winter" studies: Major uncertainties, Tech. Rep. UCRL94226, Lawrence Livermore Natl. Lab., Livermore, Calif., 1986.

Pitcher, E. J., R. C. Malone, V. Ramanathan, M. L. Blackmon $K$. Puri, and $W$. Bourke, January and July simulations with a spectral general circulation model, J. Atmos. Sci., 10, 580-604, 1983.
Ramanathan, V., E. J. Pitcher, R. C. Malone, and M. C. Blackmon, The response of a spectral general circulation model to refinements in radiative processes, $J$. Atmos. Scr., 10, 605-630, 1983.

Ramaswamy, V. and J. T. Kiehl, Sensitivities of the radiative forcing due to large loadings of smoke and dust aerosols, $J$. Geophys. Res., 90, 5597-5613, 1985.

Rodgers, C. D., and C. D. Walshaw, The computation of infrared cooling in planetary atmospheres, Quart. J. Roy. Meteor. Soc., 92, 67-92, 1966.

Roessler, D. M., and F. R. Faxvog, Optical properties of agglomerated acetylene smoke particles at $0.5145 \mu \mathrm{m}$ and $10.6 \mu \mathrm{m}$ wavelengths, J. Opt. Soc. Am., 70, 230-235, 1980.

Scientific Committee on Problems of the Environment, Environmental Consequences of Nuclear War, Report of the Bangkok Update Workshop, February 9-13, Nature, in press, 1987.

Thompson, S. L., V. V. Aleksandrov, G. L. Stenchikov, S. H. Schneider, C. Covey, and R. M. Chervin, Global climatic consequences of nuclear war: Simulations with three-dimensional models, Ambio, 19, 236-243, 1984.

Thompson, S. L., Global interactive transport simulations of nuclear war smoke, Nature, $\$ 17,35-39,1985$.

Turco, R. P., O. B. Toon, T. P. Ackerman, J. B. Pollack, and C. Sagan, Nuclear winter: Global consequences of multiple nuclear explosions, Science, 222, 1283-1292, 1983.

Washington, W. M. (Ed.), Documentation for the Community Climate Model (CCM) Version Ø, Natl. Cent. for Atmos. Res., Boulder, Colo., 1982. (Available as NTIS No. PB82-194192, from Natl. Tech. Inf. Serv., Springfield, Va.)

Washington, W. M. and G. A. Meehl, Seasonal cycle experiment on the climate sensitivity due to a doubling of $\mathrm{CO}_{2}$ with an atmospheric general circulation model coupled to a simple mixed layer ocean model, J. Geophys. Res., 89, 9475-9503, 1984.

Williamson, D. L. Description of NCAR Community Climate Model (CCMOB), NCAR Tech. Note TN-210+STR, Nat. Cent. for Atmos. Res., Boulder, Colo., 1983.

C. Covey, Atmospheric and Geophysical Sciences Division,

Lawrence Livermore National Laboratory, Livermore, CA 94550.

V. Ramaswamy, Geophysical Fluid Dynamics Laboratory, Princeton University, P. O. Box 308, Princeton NJ 08542.

S. L. Thompson, Advanced Study Program, National Center for Atmospheric Research, P. O. Box 3000, Boulder, CO 80307.

(Received September 4, 1986; revised June 8,1987 ; accepted June 10, 1987.) 